University of Redlands

\title{
TaRGeT: A Tactical Route Generation Tool for Determining an Off-Road Route
}

\footnotetext{
A Major Individual Project submitted in partial satisfaction of the requirements for the degree of Master of Science in Geographic Information Systems

by
Rachel Natalie Ginsberg

Mark Kumler, Ph.D., Chair

Fang Ren, Ph.D.

Mark Covey, M.S.
}

December 2008 
TaRGeT: A Tactical Route Generation Tool for

Determining an Off-Road Route

Copyright $(92008$

by

Rachel Natalie Ginsberg 
The report of Rachel Natalie Ginsberg is approved.
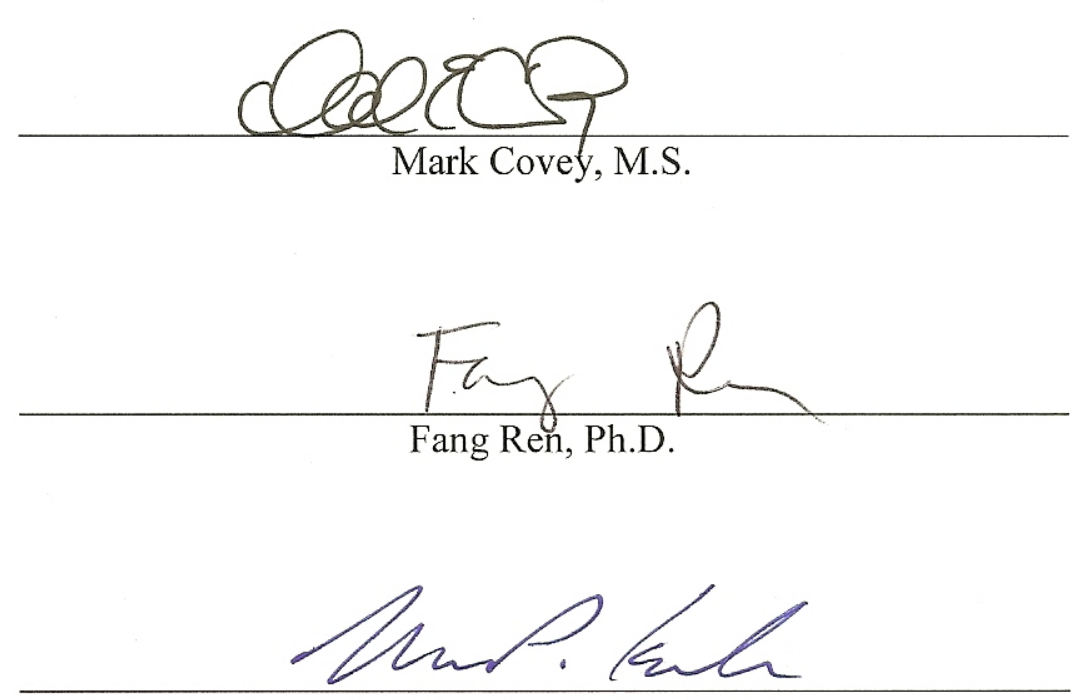

Mark Kumler, Ph.D., Chair

December 2008 



\section{ACKNOWLEDGEMENTS}

I extend my greatest thanks to my family, especially my mother Dr. Kathryn Hall and father Dr. Larry Ginsberg, for their never ending support and encouragement. To my friends who endured hearing more about GIS than they ever wanted to. I am grateful to cohort 13 (with the help of cohort 12 and 14) for making this experience both memorable and bearable.

This project would not have been possible without the help from the faculty and staff from the MS GIS program. I would like to acknowledge my advisor, Dr. Mark Kumler, and Dr. Fang Ren for being on my committee. I appreciate their involvement, patience and their enthusiasm for the subject. I would also like to acknowledge my client Mark Covey for allowing me to work on this project and being a part of my committee. I am grateful to my committee for their help and support on this project. 



\begin{abstract}
A Tactical Route Generation Tool:

Determining an Off-Road Route
\end{abstract}

by

Rachel Natalie Ginsberg

Improving training methods at military training centers will benefit preparations for combat operations and overseas operations conducted by the U.S military. One way to improve training methods is to identify off-road transportation networks for areas of military interest around the world. Using data from the Fort Irwin National Training Center combined with ArcGIS software, a model is developed to create alternate, offroad transportation routes that might be used by insurgents to avoid being detected. The methodology used in this project included creating a cost surface, using least cost path analysis, and taking additional steps in order for the final results to be compatible with other military software. Results were generated for various scenarios including routes that avoided checkpoints and steep terrain. This project was proven to be a significant tool for the client to use for training purposes. 



\section{Table of Contents}

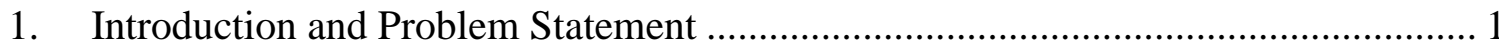

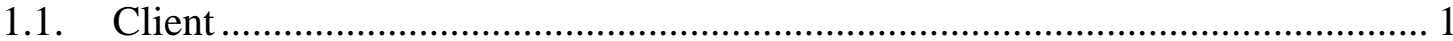

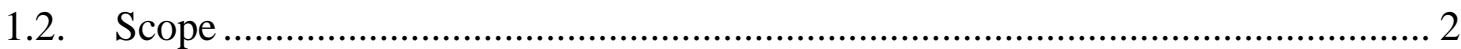

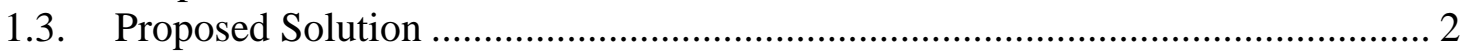

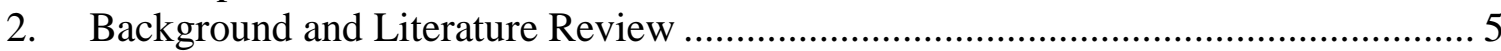

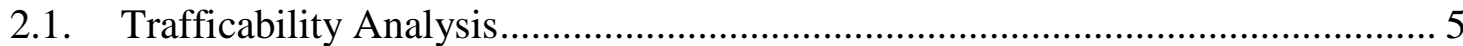

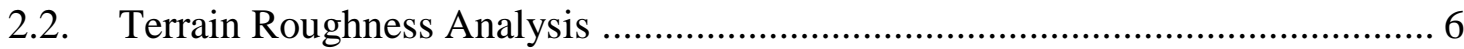

2.3. The Least Cost Path Approach .................................................................. 7

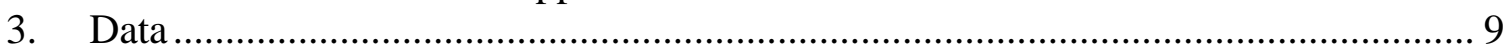

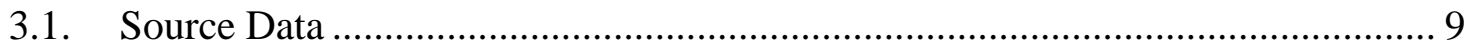

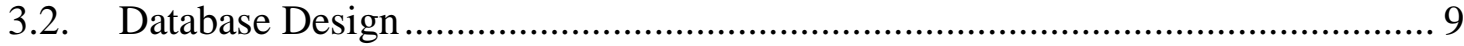

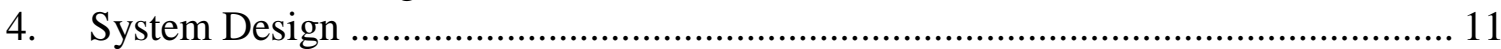

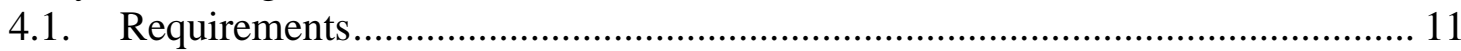

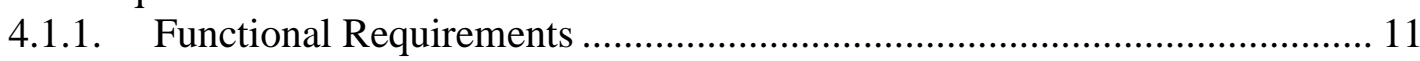

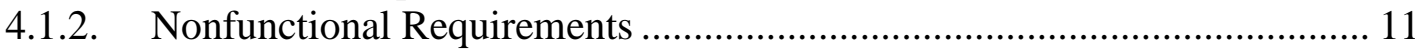

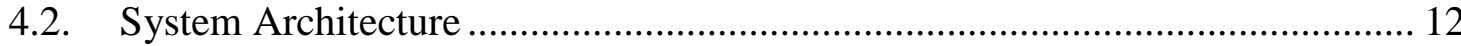

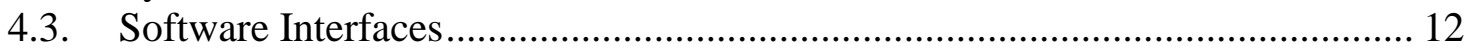

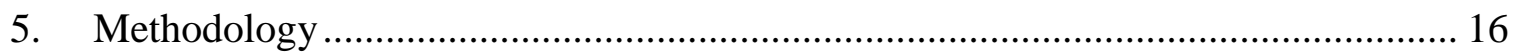

5.1. Data Validation Tasks ........................................................................ 17

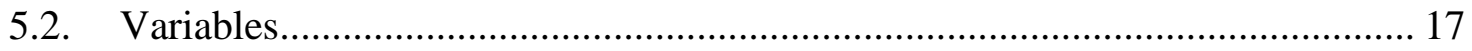

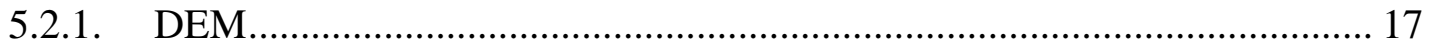

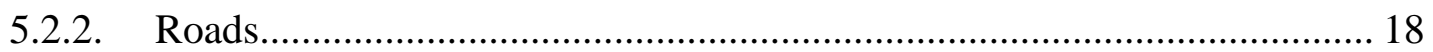

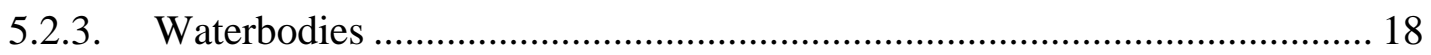

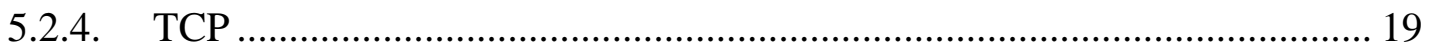

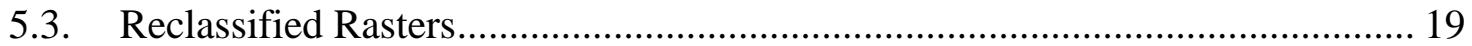

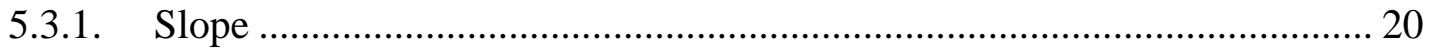

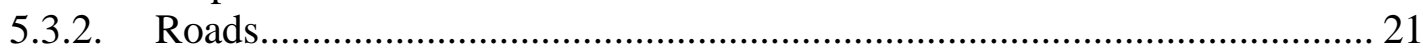

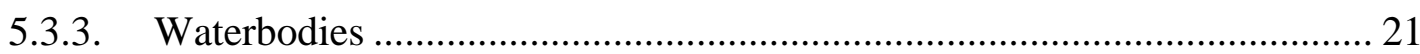

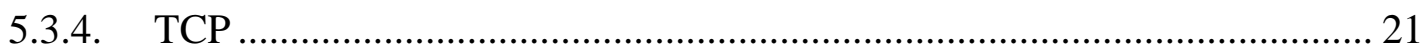

5.4. Weighted Sum ……………………………………………………….... 22

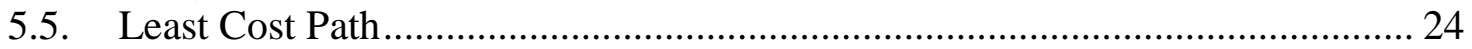

5.6. Software Compatibility ................................................................................. 24

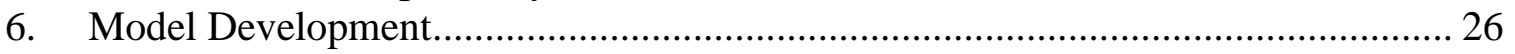

6.1. Generating Routes from a Single Point ........................................................ 26

6.2. Results with Multiple End Points................................................................... 27

6.3. Generating Routes from Multiple Start Points ............................................... 28

6.3.1. Results with a Single End Point.............................................................. 28

6.3.2. Results with Multiple End Points ............................................................. 29

6.3.3. Results with the Same End Points ........................................................... 31

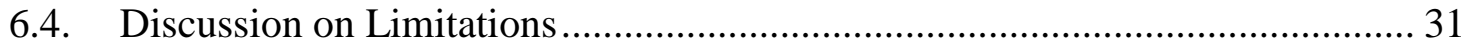

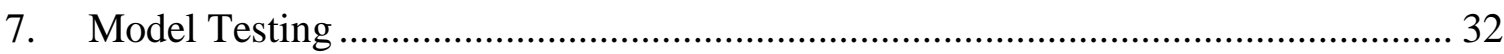

7.1. Changing the Weights of the Data Layers ........................................................ 32

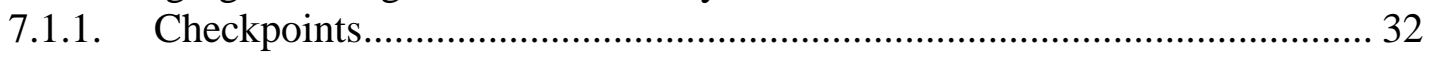

7.1.2. Changing the Slope Weight ..................................................................... 35 
7.2. Changing the Resolution of the Vector-based Datasets ................................ 36

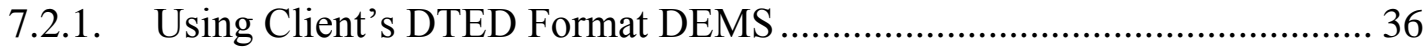

7.2.2. Using Coarser Resoultion Raster Versions of Vector Data ........................ 37

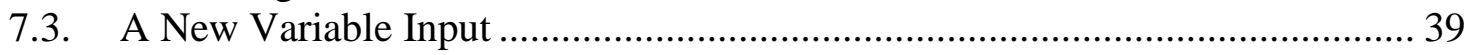

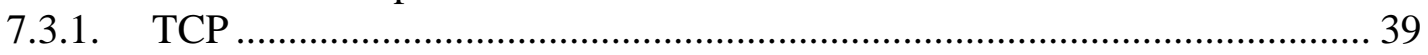

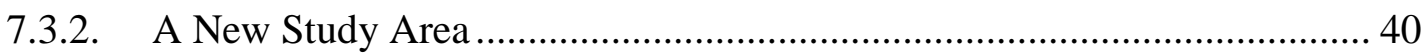

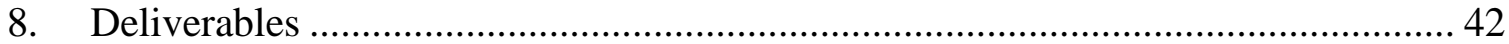

8.1. Computer Transition Testing ................................................................... 42

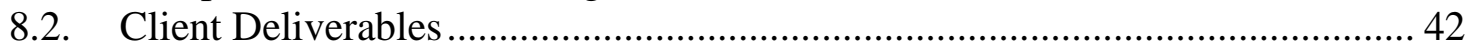

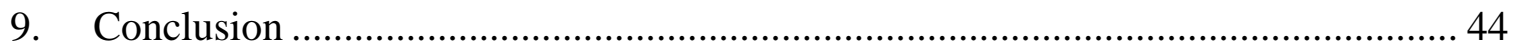

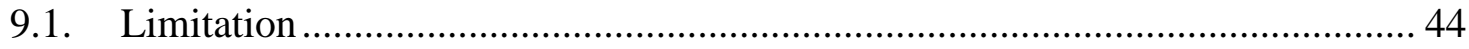

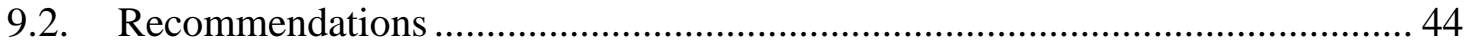

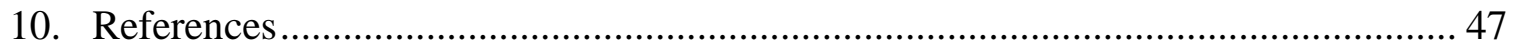

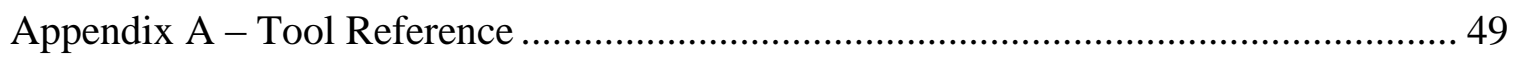

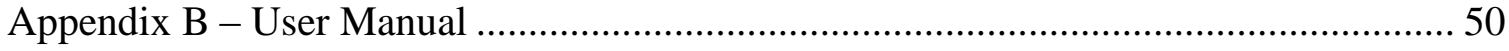




\section{Table of Figures}

Figure 1-1. Military Operations on Urban Terrain Sites at Fort Irwin Military

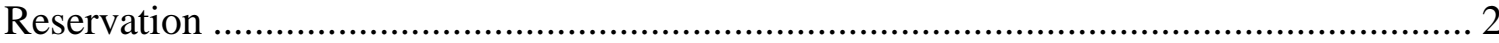

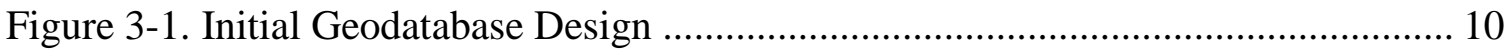

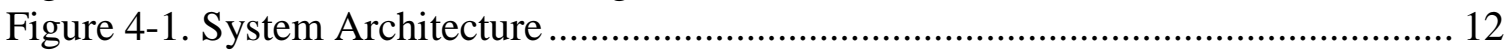

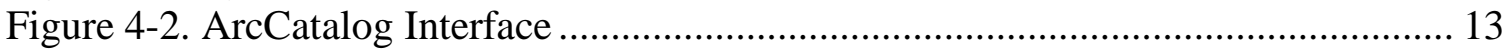

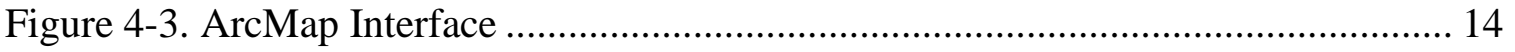

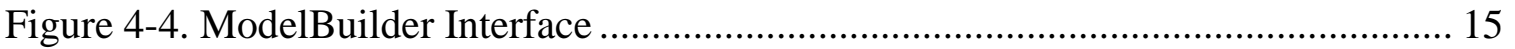

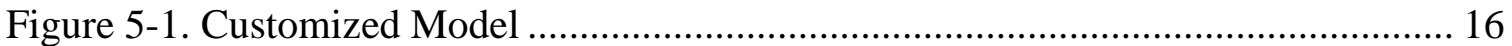

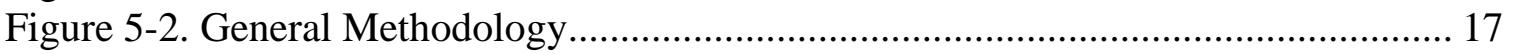

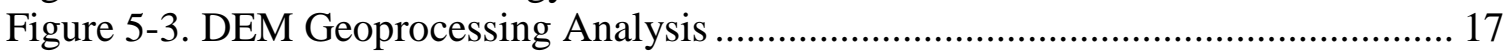

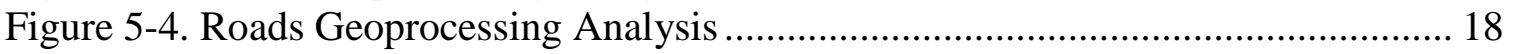

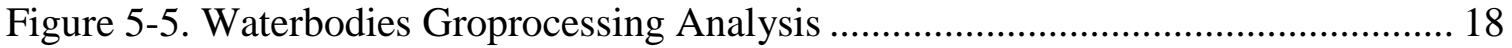

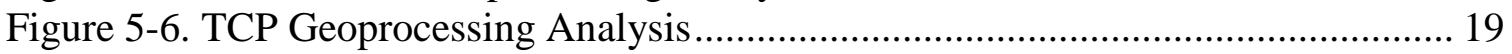

Figure 5-7. Reclassified Raster Analysis ................................................................... 20

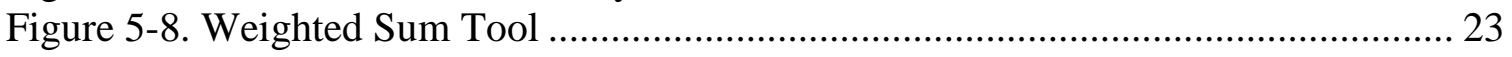

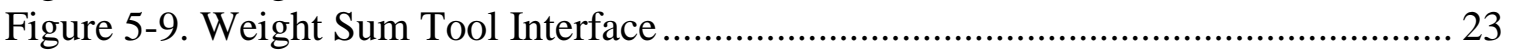

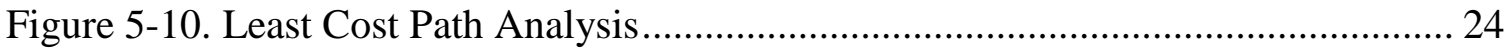

Figure 5-11. Software Compatibility Analysis .......................................................... 25

Figure 6-1. First Test from One Point to One End Point ............................................... 26

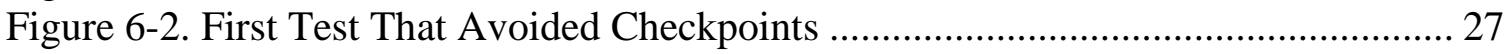

Figure 6-3. Test From One Point to Multiple End Points............................................... 28

Figure 6-4. Test with Multiple Points and One End Point............................................. 29

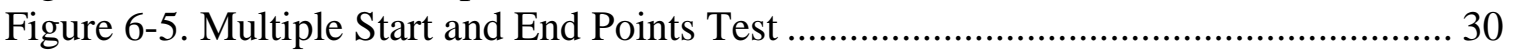

Figure 6-6. Different Multiple Start and End Points Test.......................................... 31

Figure 7-1. All Routes Between MOUT Sites that Avoid Checkpoints at 300 Meters .... 32

Figure 7-2. All Routes Between MOUT Sites that Avoid Checkpoints at 1000 Meters .. 33

Figure 7-3. Routes Avoiding US TCPs at a Greater Distance than Iraqi TCPs............... 34

Figure 7-4. Routes Avoiding Iraqi TCPs at a Greater Distance than US TCPs............... 35

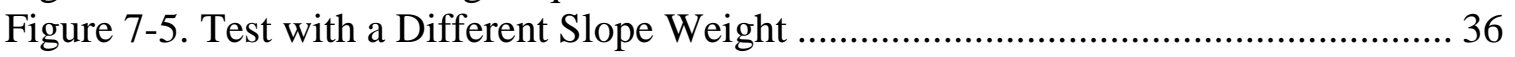

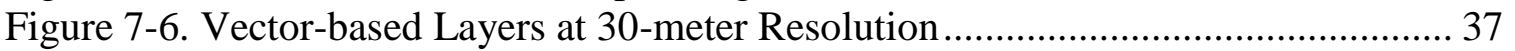

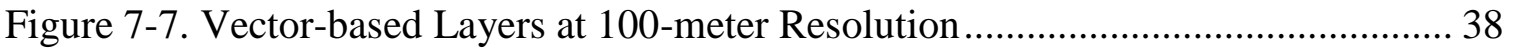

Figure 7-8. Vector-based Layers at 1000-meter Resolution ........................................... 38

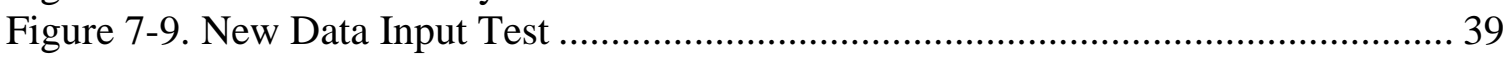

Figure 7-10. First New Study Area Test..................................................................... 40

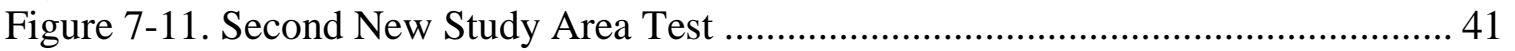

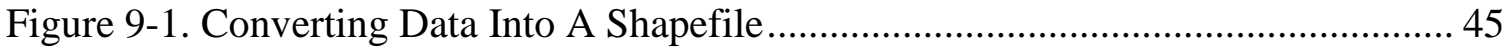




\section{List of Tables}

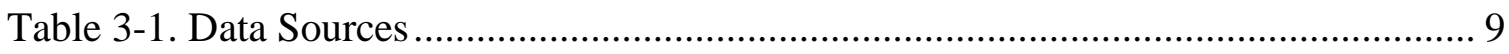

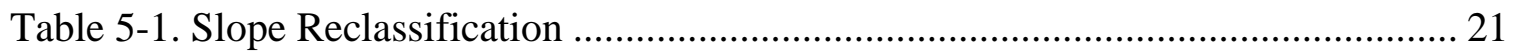

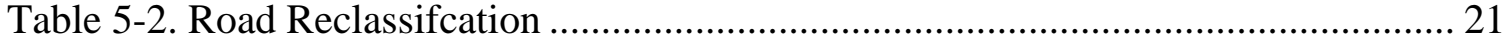

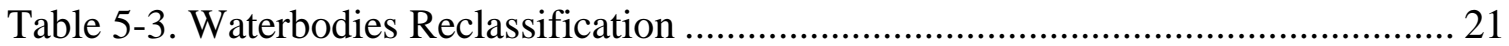

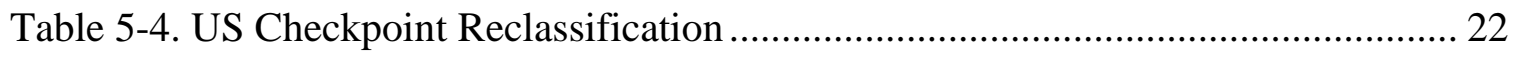

Table 5-5. Iraqi (non-US) Checkpoint Reclassification ........................................... 22 


\section{List of Acronyms}

$\begin{array}{ll}\text { DEM } & \text { Digital Elevation Model } \\ \text { DOD } & \text { Department of Defense } \\ \text { DTED } & \text { Digital Terrain Elevation Data } \\ \text { HMMWV } & \text { High Mobility Multipurpose Wheeled Vehicle } \\ \text { IED } & \text { Improvised Explosive Device } \\ \text { MCOO } & \text { Modified Combined Obstacles Overlay } \\ \text { MOUT } & \text { Military Operations on Urban Terrain } \\ \text { NTC } & \text { National Training Center } \\ \text { SIMS } & \text { Systems Integration for the Modeling and Simulations } \\ \text { TCOIC } & \text { Training Counter-IED Operational Integration Center } \\ \text { TCP } & \text { Traffic Control Points (also referred to as checkpoints) } \\ \text { TRADOC } & \text { U.S. Army Training and Doctrine Command } \\ \text { VBS2 } & \text { Virtual Battle Space 2 } \\ \text { WGS } & \text { World Geodetic System }\end{array}$





\section{Introduction and Problem Statement}

The United States military has numerous troops stationed overseas in areas of conflict and areas of potential conflict. When troops are on foreign land, they do not have the benefit of knowing the land as well as the local population. This has led to insurgents having an advantage over the United States military in some cases. In areas of conflict and areas of potential conflict, the U.S. military has set up checkpoints along the existing roads. These checkpoints exist to provide safety against insurgency. There is a wide range of tactics that insurgents' use, which include improvised explosive devices (IEDs), ambushes, suicide bombers, and attacks on security forces. Insurgents have often done their own reconnaissance, and with their superior knowledge of the local landscape, may be able to avoid these checkpoints without detection.

The Department of Defense (DOD) has training centers globally, in addition to the centers within the United States. More specifically, the U.S. Army has training centers within the United States that simulate foreign lands. The National Training Center (NTC) at Fort Irwin is used to represent areas of conflict or expected conflict and is used for training purposes for future operations. Troops in areas of conflict are noticing that insurgents are avoiding the established transportation network where there is a U.S. military presence, and are instead creating an off-road network of routes to get to their destinations. In some situations, insurgents are also avoiding the local military and police checkpoints. In any military situation - whether training or the physical battlefield - these routes should be recognized.

Along with using the NTC at Fort Irwin as a physical training ground for the troops, the military also uses gaming and virtual simulations to replicate conditions where military personnel are deployed. One software package that the military uses is Virtual Battle Space 2 (VBS2). VBS2 is an advanced virtual environment training system used for military mission rehearsal and tactical training purposes. VBS2 accepts ESRI format shapefiles for the data. One shapefile contains the existing transportation network. The transportation network contains roads that vehicles must stay on in a simulated mission using strictly artificial intelligence in VBS2.

Part of strategic military planning is to provide troops with the best training possible before deployment. Training includes finding ways to predict where the enemy may move in order to escape detection. One way to provide better training is to include plausible off-road routes that an insurgent might take in order to avoid detection. The objective of this project is to produce a tool that creates shapefiles to be used with VBS2 software which contain plausible off-road routes that avoid checkpoints.

\subsection{Client}

The client for this project is the U.S. Army Training Counter-IED Operational Integration Center (TCOIC), with Mark Covey as the point of contact. Mark Covey is the Director of Systems Integration for the Modeling and Simulations (SIMS) Directorate for the TCOIC. The SIMS Directorate will use this application in a training environment and apply it to military areas of interest around the world. The immediate intention is to apply this project to the current situation in the Middle East. 
TCOIC falls under the administrative command of the U.S. Army Training and Doctrine Command (TRADOC) and takes operational direction from the Joint IED Defeat Organization (JIEDDO). It is responsible for building the future Army by recruiting, educating, and training the Army's soldiers. TRADOC's priorities include supporting the nation during wartime "while simultaneously anticipating solutions to the challenges of tomorrow" (TRADOC, 2008). TRADOC includes fifteen installations with twenty-seven specialized schools throughout the United States.

\subsection{Scope}

The scope of this project was tested on a specific area defined by the client. The project focused on data from the National Training Center at Fort Irwin, which is located in the Mojave Desert of California. Fort Irwin Military Reservation is used to represent any area of conflict or expect conflict and for this project will represent Iraq by having five military operations on urban terrain (MOUT) sites, as displayed in Figure 1-1.

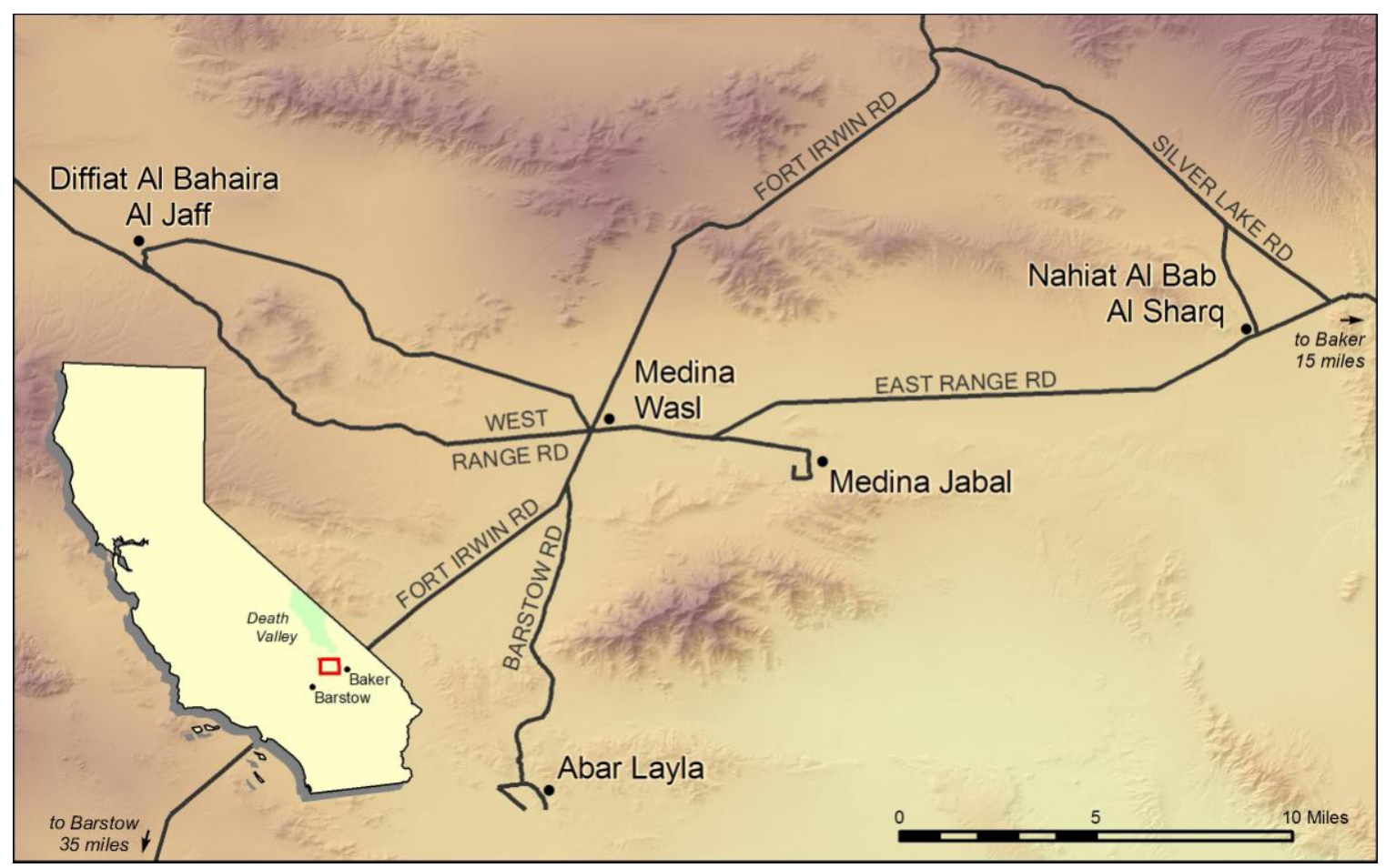

Figure 1-1. Military Operations on Urban Terrain Sites at Fort Irwin Military Reservation

\subsection{Proposed Solution}

The proposed solution for this project was to create a tactical route generation tool. This tool was a customized toolset that generated off-road routes that linked to a pre-existing transportation network. The off-road routes were plausible routes that an insurgent might take in order to avoid the military presence of checkpoints on the existing roads. The final output of the toolset was a shapefile that contained both existing roads 
and off-road routes to be used for training purposes. The shapefiles' geometry had to consist of polyline features to be compatible with the VBS2 software.

The goal of the solution was to have the toolset be compatible with current military software. The software used to create the toolset was built using ArcGIS software with an ArcInfo 9.3 license. Within the ArcGIS software, the ModelBuilder application is used to automate geoprocessing tasks through the use of creating, editing and managing models. To create a customized model, tools from existing toolboxes with ArcToolbox would be used to create a string of processes to generate the shapefile. This customized model was referred to as model tool that was created in ModelBuilder. The final models were stored within a toolset within a toolbox.

The series of processes within the model was created based on the concept of a least-cost path. A least-cost path is a path between two points that has a minimum total cost, where the cost is a function of multiple criteria defined by the user. In this project, the path represents a plausible route that an insurgent might take to avoid military checkpoints.

In a military training environment, including the VBS2 virtual environment, plausible off-road routes should be recognized. The tactical route generation tool described in this report is a valuable resource for identifying such routes. 



\section{Background and Literature Review}

The purpose of this project was to find a way to generate plausible off road routes that an insurgent might take in order to avoid military checkpoints on existing roads. The approach used to accomplish this was to conduct trafficability analysis to create a cost surface between pairs of points, considering not just the existing road network, but all off-road options as well. The cost surface contained several variables that affected how a vehicle travels through an area. One of those factors included an analysis of terrain ruggedness. Each factor used in the trafficability analysis generated a cost surface. The cost surface has a raster format, meaning every cell in the raster is associated with a travel cost value. These cost surfaces were used to represent the cost of travel through each cell, and then to calculate the cumulative costs of travel through the area of interest. Finally, the least cost path (or an off-road route for this project) was generated from the cost surface.

\subsection{Trafficability Analysis}

Trafficability commonly refers to the ability a vehicle can maneuver through a specific area or region of terrain. The U.S. military has done significant work on this subject, but it has also been applied in civilian planning. The movements of vehicles and troops over various terrain has been one of the military's top concerns.

Donlon and Forbus (1999) focused on the military’s trafficability problems. Most military terrain analysis begins with map data from topographic maps. Overlays that outline areas with uniform characteristics of the terrain were created on transparent sheets and placed on top of topographic maps. Each overlay represented a different terrain factor that would affect a vehicle's ability to travel, such as slope, vegetation, hydrogeology, and soil type. This overlay process allowed an "analyst to compute estimates of vehicle speeds for each region, based on the vehicle capabilities" (Donlon \& Forbus, 1999, p. 4).

Trafficability domain theories involve knowledge of terrain, how terrain effects any movement, vehicles' capabilities for on and off road mobility, and how the terrain affects the vehicles movements. Donlon and Forbus constructed domain theories that included those areas with knowledge of how digital representation of the terrain is determined. They assert that "trafficability is a binary relationship between the terrain and the vehicle" and it can be represented qualitatively or quantitatively (Donlon \& Forbus, 1999. p. 5). The ability of a vehicle to effectively travel through an area is expressed qualitatively and the effect the terrain has on that movement is expressed quantitatively. The results from their study were consistent with the U.S Army's methods. Their method helped reduce the time needed to calculate the same analysis. Further testing with tougher terrain of this model was suggested, along with incorporating information about soil type and surface configuration.

A trafficability analysis engine was developed by Slocum, Surdu, Sullivan, Rudak, Colvin, and Gates (2003). This prototype was developed to provide a quicker method to analyzing the results of the U.S. Army's Modified Combined Obstacles Overlay (MCOO), which is a manual process. The MCOO classifies the terrain into either "go", "slow-go", or "no-go" categories. Many factors that could affect trafficability were not included in the MCOO process, but this prototype includes several geographic factors, including "location, vehicle type, off-limits terrain, water, weather, soil, land use, 
topography, vegetation and roads" (Slocum, Surdu, Sullivan, Rudak, Colvin, \& Gates, 2003). The engine was also designed to be user friendly. Trafficability is computed by calculating each geographic factor individually before being used in the final trafficability analysis. The results of this system were determined to be very close to the results from the manual analysis. The individual geographic factors needed more development, but the speed of this engine was a major improvement.

A GIS-based terrain mobility model that included the optimization of off-road roads is another trafficability concept developed by Suvinen, Saarilahti, and Tokola (2003). The influence of a machine, terrain, road, and weather was used to generate a cost surface. They state that terrain mobility depends on the soil's ability to resist forces applied by a rolling wheel, other properties of the terrain, the machine's physical measurements, and the number of wheels. Other obstacles included in this model included tree coverage, soil moisture, large rocks, steep slopes, and the presence of power cables. Each parameter was calculated together to establish a terrain cost surface. A sample area was used to calculate a cost grid and different cost surface alternatives could be generated when information about the objects are changed. Alternative routes could be generated from different conditions from a regular costpath analysis. The results of this study have "shown that useful parameters can be found for national level digital maps for supporting off-road analysis" (Suvinen et al., 2003, p. 257).

The Trafficability Evaluation System (TES) was developed by Eichrodt (2003) in order to provide better trafficability analysis of forestry equipment over the terrain of Switzerland forests. The concept of TES was determined by terrain analysis models, modeling disturbances, and having the maximum vehicle gradeability calculated. The study consisted of three test scenarios to determine the model's plausibility. The first test compared the trafficability limits of a wheeled vehicle to those of a tracked vehicle. The second scenario tested "the effect of tire inflation pressure on the extent of trafficability" (Eichrodt, 2003, p. X). The last test compared trafficability from two different dates that had different water contents do to the temporal component. Eichrodt concluded that TES was able to provide acceptable accuracy for predicting water content and soil strength for vehicle mobility.

\subsection{Terrain Roughness Analysis}

Terrain roughness is commonly one of the major factors used in accessing trafficability especially for path planning. This analysis illustrates how irregular the elevation is between adjacent cells in a digital elevation model (DEM) of an area. The military has used this type of analysis for their mapping. Crawford (2008) noted that there are many models that incorporate this analysis, but are created for a specific purpose. These models are often not flexible and are simplified, leaving the model incomplete.

Hoffman and Krotkov (1989) argued that a single parameter for the surface analysis of terrain in insufficient to determine the surface roughness. Their study presented "a vector measurement of terrain surface roughness that includes amplitude, frequency, and autocorrelation components" (Hoffman \& Krotkov, 1989, p. 105). Their method incorporated using Fourier analysis, which studied geological surface irregularities that were measured from a fixed point of elevation that had internal difference from seven centimeters to three meters of elevation. "This method measures 
roughness along specific directions of a surface and includes amplitude, frequency, and autocorrelation terms" (Crawford, 2008; Hoffman \& Krotkov, 1989, p.107).

Hoffman and Krotkov wanted the measurement to also be intrinsic to the surface, but the Fourier analysis does not include the intrinsic roughness measurement of the surface. They replaced the global measurement of the Fourier analysis with their own local measurement for measuring the intrinsic values. Hoffman and Krotkov stated that they developed "a technique for estimating the local, intrinsic roughness of terrain" that identifies regions that have a relative smooth surface when applied to elevation maps (Hoffman \& Krotkov, 1989, p. 112).

A Spatial Analyzer Model was developed to analyze geomorphometric characteristics of geo-images. This model generates surface layers that include the topographic ruggedness and the drainage density. The surface layers were used in the overlay function of the model "to make topographic overlays which can be used to identify areas of risk" (Moreno, Levachkine, Torres, \& Quintero, 2003, p. 645). The terrain ruggedness layer used the terrain ruggedness index (TRI) measurement to indicate the elevation difference between the adjacent cells in a digital elevation model. The process computes the difference in elevation values from a single cell to the eight surrounding cells. After the terrain ruggedness index is derived, it is then compared to the pre-determined scale for classification. The study resulted in significantly decreasing the time and effort needed to quantify terrain characteristics.

\subsection{The Least Cost Path Approach}

The first step to creating a least cost path is to create a cost surface. A cost surface is produced by classifying at least one raster, which contain data that affects the cost of travel, such as elevation, vegetation, soil composition, and a wide variety of other factors. Each factor can then be given a relative weight and the cost of travel through each cell of the raster between points can be calculated. This will determine where the least cost path is.

Using geographic information systems for transportation purposes has been employed since interest in GIS began in the 1960s (Goodchild, 2000). Tobler (1993) was concerned about developing a cost surface to include various costs in a hilly terrain. Since transportation systems are complex, Tobler wanted to develop a more realistic time cost between places. Tobler developed a basic equation for walking in a hilly landscape by calculating the traveler's velocity from the slope of the terrain. He envisioned the advancement of geographic information systems in a way that would incorporate complex spatial analysis by combining continuous cost surface approaches with network models. Since then, advances in software have enabled more complex terrain analysis.

Lee and Stucky (1998) made an advancement in the least cost path analysis area by using viewshed information (area of land this is visible from a fixed point) calculated from digital elevation models. Their analysis included four different types of paths. The strategic path and hidden path have applications for military surveillance, reconnaissance, and special forces operations. In order to create more realistic models, Lee and Stucky noted that other factors should be considered, since they only used digital elevation models. They concluded that a vector data model may be more helpful than a raster data model. 
Least cost path analyses are used extensively in applications such as highway and canal planning. Collischonn and Pilar (2000) used least cost path algorithms to find best paths based on known topography, using two points from either end of a canal or highway (a linear feature) and a function involving slope, distance, and cost-of-passage to develop an algorithm. This is just one example of how the basic methodology of least cost path analyses is used in complex problems.

An extension to the least cost path algorithm was developed by Yu, Lee, and Munro-Stasiuk (2003). They included the elements of distance, anisotropic (directionally dependent) costs, and the presence of bridges and tunnels along the paths. This approach created a more practical method for roadway planning using the least cost path algorithm. However, there were limitations in this study. Limitations included further exploration of a variety of methods for weighting different parameters on this algorithm. Other limitations include geological conditions and the curvature of terrain surfaces.

Balstrom (2002) developed a friction-based cost surface. He was interested in developing a procedure to find the most efficient walking route between a number of points where roads and walking paths did not exist. The route used a digital elevation model to determine walking times based on the slope of the terrain and river crossing as part of the friction-based cost surface. A major limitation that Balstom noted was that the slope was accounted for in only one direction. This meant that entering a cell as either traveling uphill or downhill was not possible using the software that was available, thus limiting a traveler's decision to choose to travel along a slope.

Wood and Wood (2006) developed a new approach to least cost path analysis that involved a caloric cost model for pedestrian travel across terrain. This approach analyzed paths of least caloric expense for new methods of terrain visualization. They developed a network consisting of the shortest path, least cost path, and caloric terrain calculations. The weights they assigned to each edge along the path representing caloric cost included a metabolic equation involving factors such as traveler's sex, age, weight, height, and speed. This computation tool was developed for archeological applications, but there are military applications to evaluate enemy off-road routes on foot. 


\section{Data}

The client provided the initial datasets. These datasets contain the same attribute information and formats as all other data that the client has and may use for future use with this project. The development and future use of this project is based on these standards and formats. The datasets also included additional information for the area of interest that was not used for the project. The data was delivered in a geodatabase and the necessary datasets were placed into a new file geodatabase.

\subsection{Source Data}

The datasets used in the geodatabase are listed in Table 3-1. Many of the dataset names were provided by the client and preserved in this project for the ease of transition back to the client. These datsets are not all inclusive, but were simply a sample of datsets that can be used. Additional features that can affect trafficbility may be included for future use (see chapter 9).

Table 3-1. Data Sources

\begin{tabular}{|l|l|l|l|}
\hline \multicolumn{1}{|c|}{ Dataset } & \multicolumn{1}{|c|}{ Description } & \multicolumn{1}{c|}{ Source and Date } & Data Geometry \\
\hline DEM & Digital Elevation Model & USGS, 2008 & Raster \\
\hline Road & $\begin{array}{l}\text { Existing transportation } \\
\text { network }\end{array}$ & TCOIC, 2008 & Polyline (Vector) \\
\hline TCP & $\begin{array}{l}\text { US and Iraqi traffic control } \\
\text { points }\end{array}$ & TOCIC, 2008 & Point (Vector) \\
\hline WaterBodies & Large water bodies & USGS, 2004 & Polygon (Vector) \\
\hline Info & MOUT sites & TCOIC, 2008 & Point (Vector) \\
\hline
\end{tabular}

All data provided by the client were in the WGS 1984 projection. Data that came from other sources were reprojected to match the projection of the client's data. It was agreed upon with the client that a new DEM and water dataset were needed. Several DEM's were provided by the client, but a higher resolution DEM was found at the USGS site. The water dataset provided by the client was empty and another source was used.

\subsection{Database Design}

The file geodatabase contained a feature dataset, the DEM, and a toolbox. There was no manipulation of the data attributes before running the data in the customized tool. The original tables and attributes in the checkpoint feature class determined the necessary queries to be performed during geoprocessing. The customized toolbox was designed with a selection of attributes for the checkpoint data layer. The uses for a feature dataset, feature class, and toolbox are listed below. 
Feature Dataset: Vector data was contained within a feature dataset in order for the projection to remain consistent.

Feature Class: The feature classes contained all the input data which included a DEM, checkpoints (TCP, see Table 3-1), roads, MOUT sites (info, see Table 3-1), and hydrology. The DEM was in raster format. The checkpoint data had to have an attribute field that contained the type of checkpoint it was. The field was called TCP and contained US as the needed attribute name. Any other attribute names were considered to be non-US. This project used Iraq as the scenario and the nonUS attribute name was IRQ. MOUT site data was used to create start and endpoints.

Toolbox: This toolbox contained the toolset with all the customized models. The full model had all the processes needed to create the output, and was designed with the input parameters to be the same as the feature classes. It also performed a query based on the checkpoint data attribution and created a feature class that contained the existing road network and new off road routes.

The initial file geodatabase structure is seen in figure 3-1.

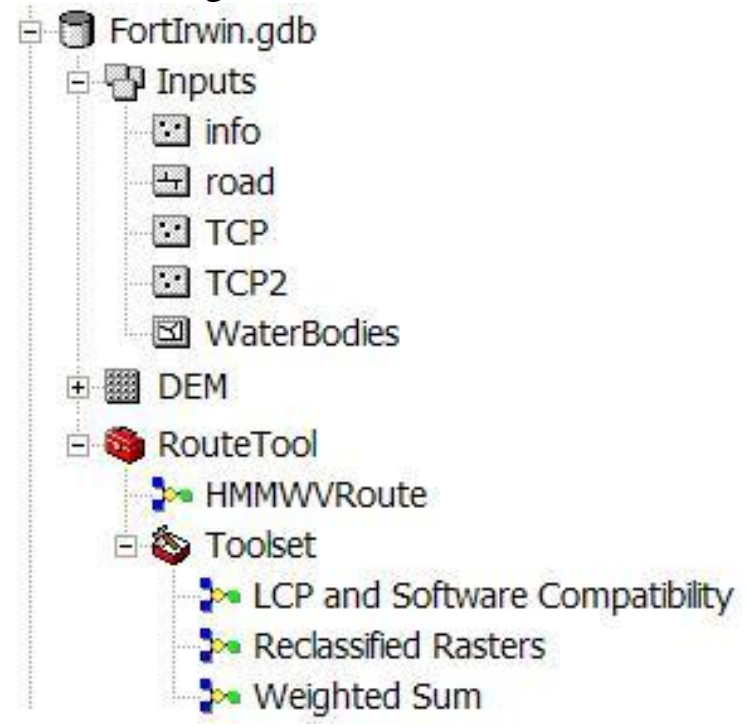

Figure 3-1. Initial Geodatabase Design 


\section{System Design}

System design requirement were established through discussions with the client. The overall system design included requirements, the system architecture, and software interfaces.

\subsection{Requirements}

The proposed solution of the project had several functional and nonfunctional requirements.

\subsubsection{Functional Requirements}

There are sets of functional requirement. One set is for the entire project and the other set is specifically for the model tool component that was developed.

The overall project system shall:

- visually display the results.

- produce a shapefile that contains a least cost path that represents an off-road route.

The model tool shall:

- incorporate no more than the data that was provided for the client as possible parameters for the tool.

- have the following feature classes as input parameters:

- Digital Elevation Model

○ Checkpoints

○ Start point

E End point

- Hydrology

- have the directory and name of the final output as a parameter.

- create the slope from the DEM.

- create results that restrict the final path to areas where the slope of the DEM meets the capabilities of a high mobility multipurpose wheeled vehicle (HMMWV).

- create buffer rings to represent the effective range of a weapon around a TCP feature.

- convert all vector data to raster data.

- reclassify all raster data into new values between one and nine.

- reclassify the raster data with the new values according to the client's requests.

- add all reclassified rasters together, which will result in a cumulative cost surface.

- run a cost distance tool and include the cost surface raster and start point as the inputs.

- run a cost path tool and include backlink, cost distance, and end point as the inputs.

\subsubsection{Nonfunctional Requirements}

The nonfunctional requirements of the overall system shall:

- be built using ArcGIS 9.3 software. 
- be built using ArcGIS ModelBuilder.

- be able to run on an ArcInfo license.

- delivered in a file geodatabase.

- contain a user's guide that describes the requirements for the route generation tool to work.

- be user friendly with clear labeling of the input data and final output path fields.

- produce logical results to be determined by the client.

\subsection{System Architecture}

The system architecture is shown in Figure 4-1. The components of this system began with data input, which were used to generate a cost surface or as a source or destination point. Once all the data was collected, it was reviewed and edited in order to comply with the database design. All the data was stored within a file geodatabase. The geodatabase also contained a customized toolbox that contained the tool to generate a new route based on the data inputs. The next step was to run the tool, which used least cost path analysis to produce a feature class as an output. The feature class was then reviewed for post editing tasks including converting the feature class into a shapefile. The last step of this system was to put the shapefile as an input into the VBS2 software, which was used by the client.

Data

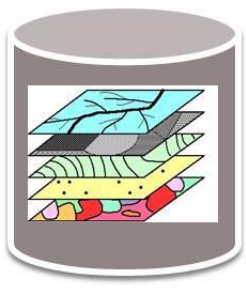

\section{Least Cost Path} Analysis

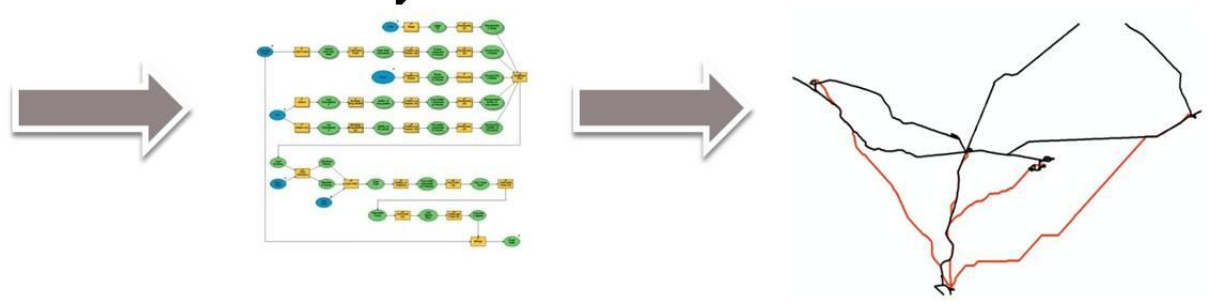

Battle Simulation

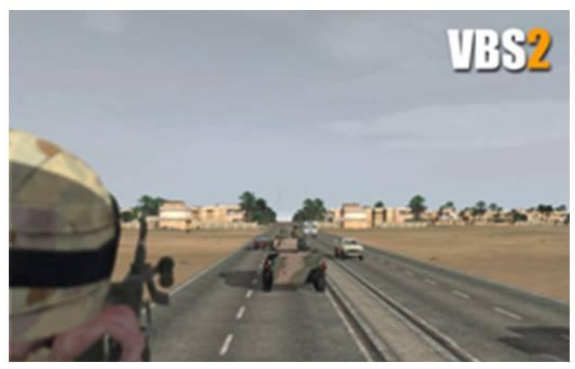

\section{Evasive Routes}

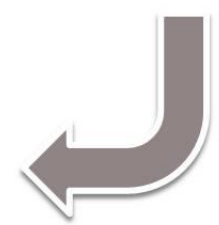

Figure 4-1. System Architecture

\subsection{Software Interfaces}

Several individual software components made up the system and satisfied the requirements determined by the client. These components included the software interfaces that were needed in the system: 
ArcCatalog: This was used to organize and maintain all data inside a file geodatabase. The ArcCatalog interface can be used to preview data in either geography or table format (see Figure 4-2).

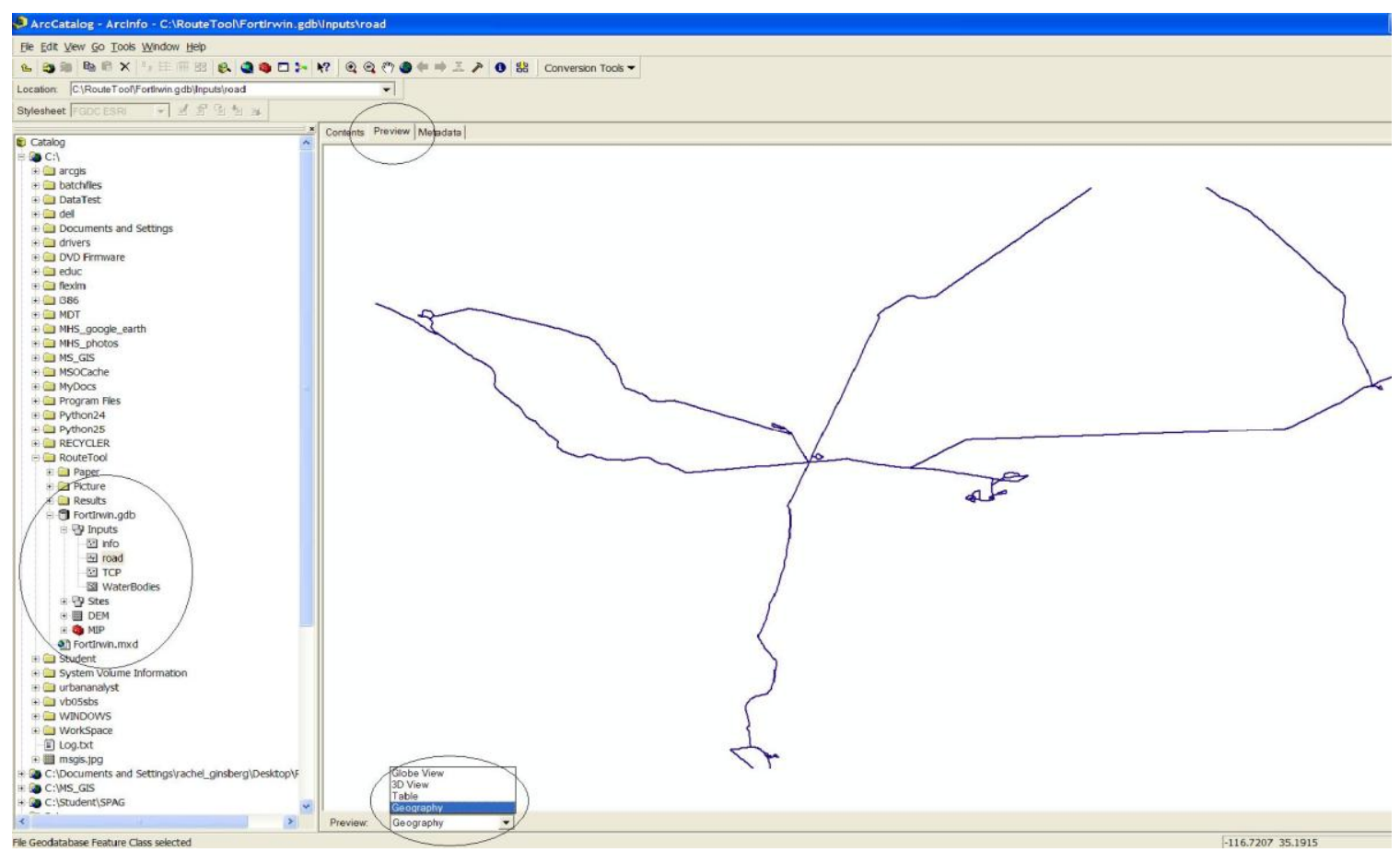

Figure 4-2. ArcCatalog Interface

ArcMap: This was used to display and edit data during post-model editing. Figure 4-3 shows the ArcMap interface with data layers on the right, the editor toolbar on top and main window being the map layout. 


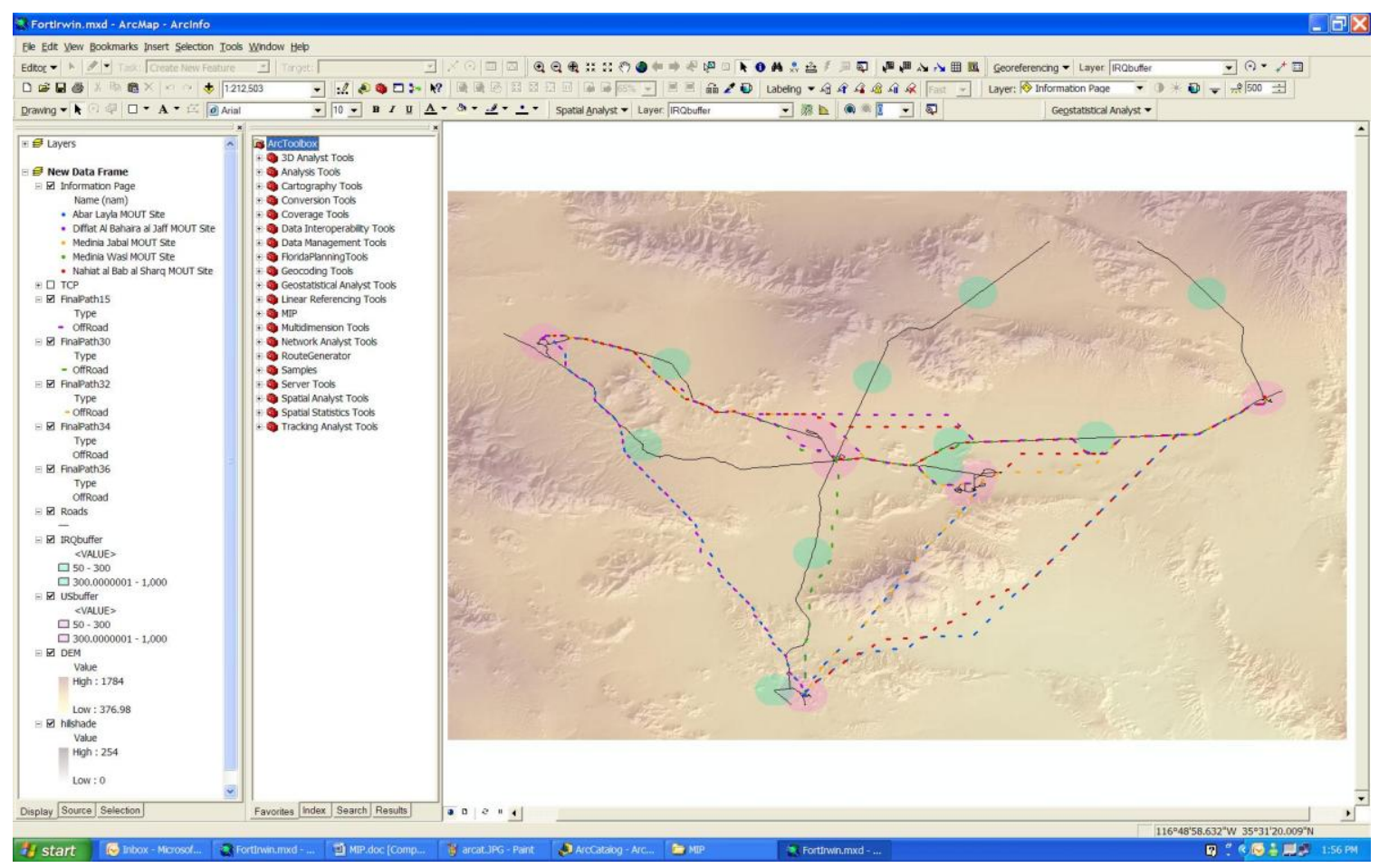

Figure 4-3. ArcMap Interface

ArcToolbox: This was used to locate the existing tools needed for the customized tool. The ArcToolbox interface can be open in either ArcMap or ArcCatalog.

ModelBuilder: ModelBuilder was used to create a model within a toolbox. This was used to customize a tool and for the user to manipulate the tool in future uses. The interface allows existing tools from ArcToolBox and data inputs to produce new datasets as seen in Figure 4-4. This figure will be presented again, reformatted for legibility in the following chapter. It is included here just to illustrate its appearance within ModelBuilder. 


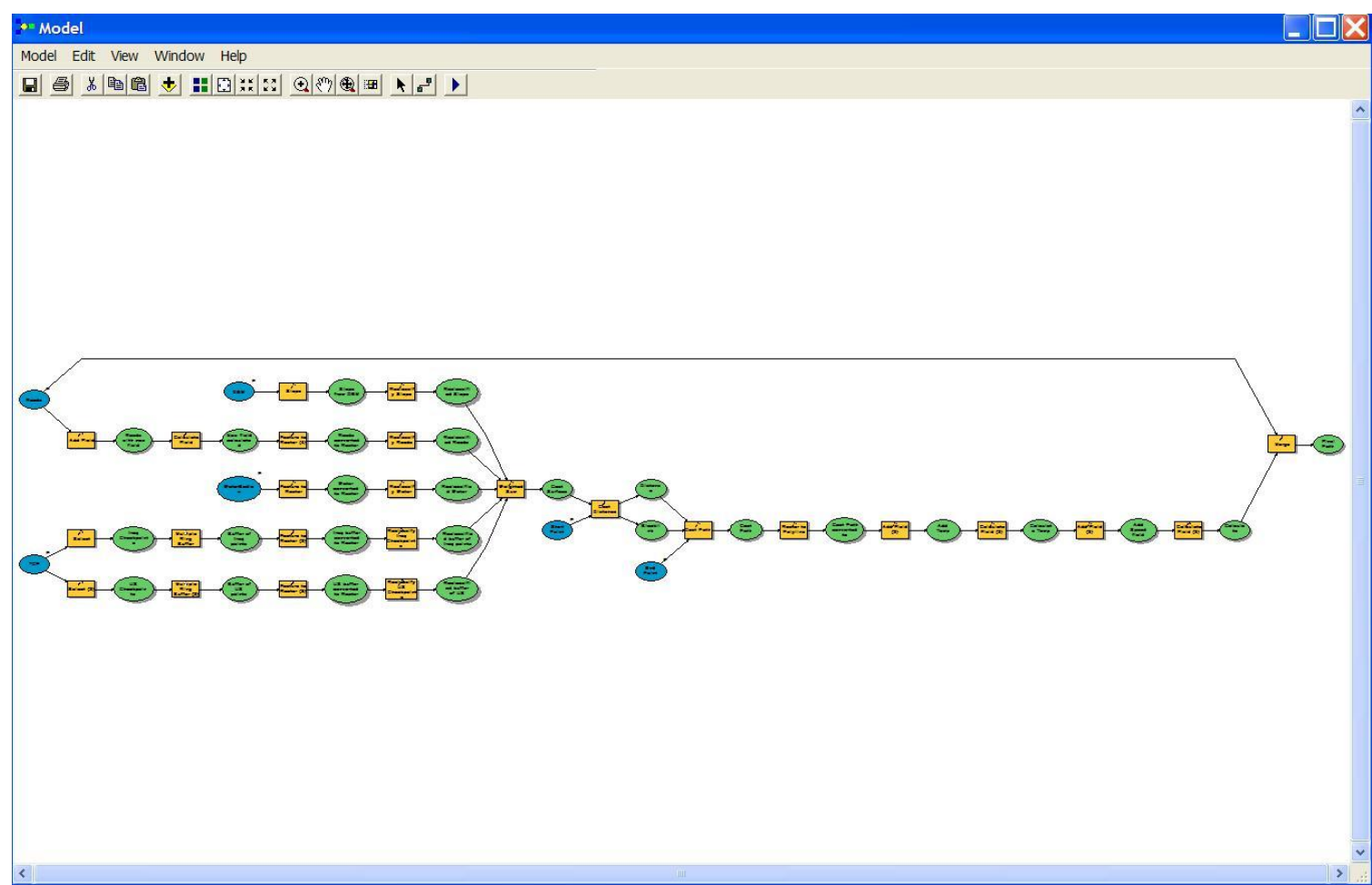

Figure 4-4. ModelBuilder Interface

Virtual Battle Space 2 (VBS2): This was the final system component that the user will need for military training purposes. The input for this system is the final shapefile that was created in post editing. 


\section{Methodology}

The approach used to create the customized model was to use ModelBuilder to string together existing tools from the ArcGIS ArcToolbox. The complete model is illustrated in Figure 5-1.

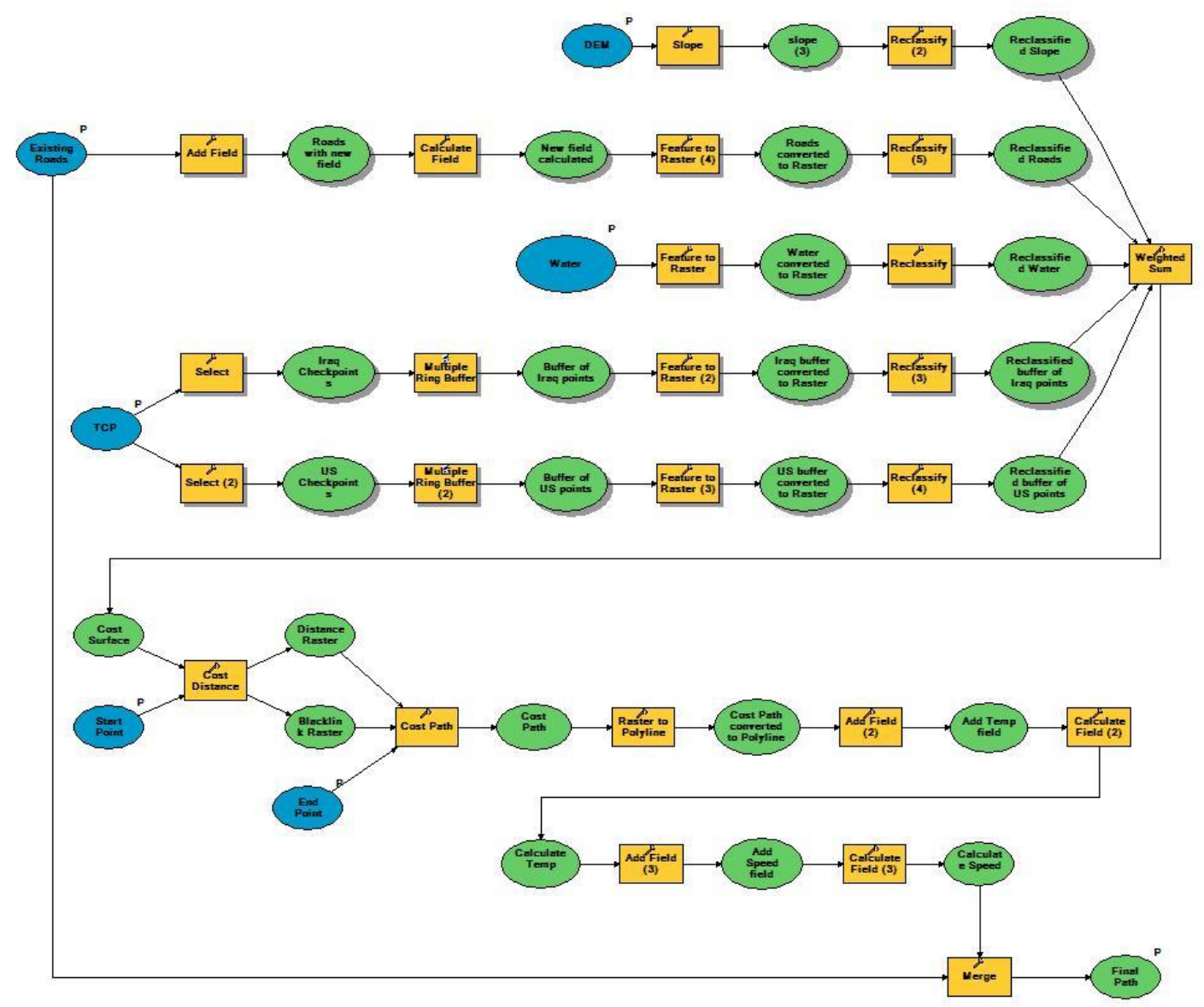

Figure 5-1. Customized Model

This model was broken down into six general steps (see Figure 5-2). This chapter describes each step. 


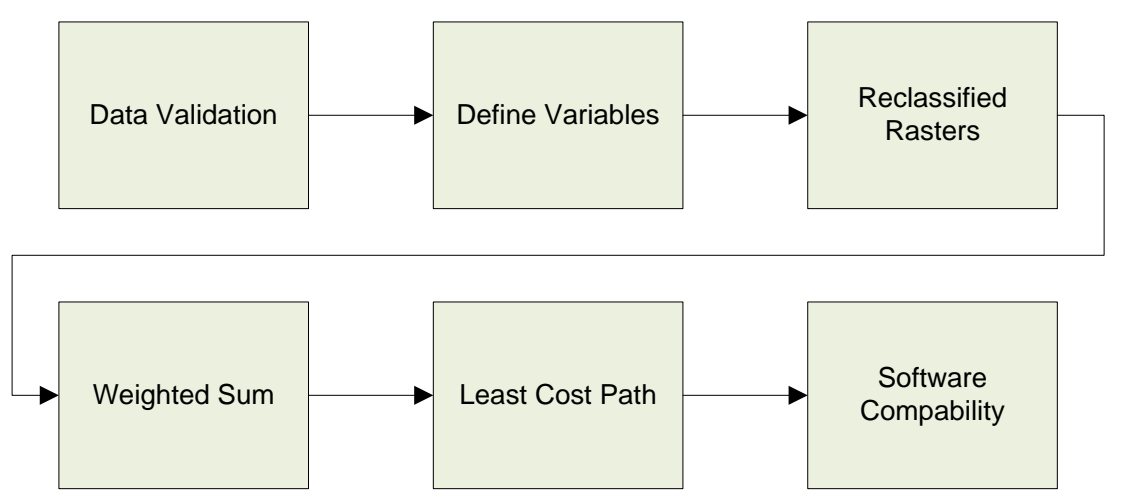

Figure 5-2. General Methodology

\subsection{Data Validation Tasks}

Before running the model, the following few tasks were conducted:

1) All file paths to the data were identified.

2) All data were in the same projection.

3) All input datasets contain data features (no empty datasets).

4) Created two feature classes from the MOUT sites data: one that contained the start point and another that contained the end point.

5) The checkpoint data contained an attribute called TCP and the only values were either US or IRQ.

\subsection{Variables}

The model requires several variables for performing the spatial analysis. This section presents the variables and describes how they affect the cost of travel to make a cost surface. All variables were converted to raster surfaces before the cost surface was calculated.

\subsubsection{DEM}

The DEM was used to calculate the slope for each cell in the study area, which affected a vehicle's ability to pass through the cell. There is a maximum limit to which vehicles can transverse a given slope. The only geoprocessing tool needed was the Slope tool. The result was a raster with each cell containing a slope value between 0 and 90 degrees. Figure 5-3 shows the geoproessing analysis for the DEM data.

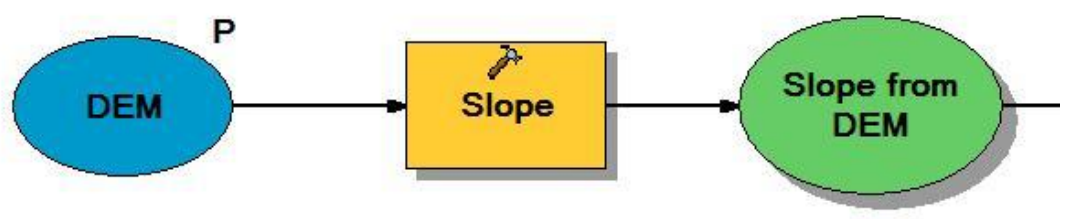

Figure 5-3. DEM Geoprocessing Analysis 


\subsubsection{Roads}

The roads dataset consisted of the existing transportation network. The existing roads are the main means through which a vehicle would travel. The roads data geometry was in vector format and needed to be converted into a raster.

The first geoprocessing tool needed was the Add Field tool. This created a field called temp (for temporary). The next tool, Calculate Field, calculated the temporary field to the value of one. The Feature to Raster tool was then used to convert the vector data to a raster format based on the newly added field. The result was a raster that contained the value of one where there were roads, and the value of no data for all other cells. The roads geoprocessing analysis is shown in Figure 5-4.

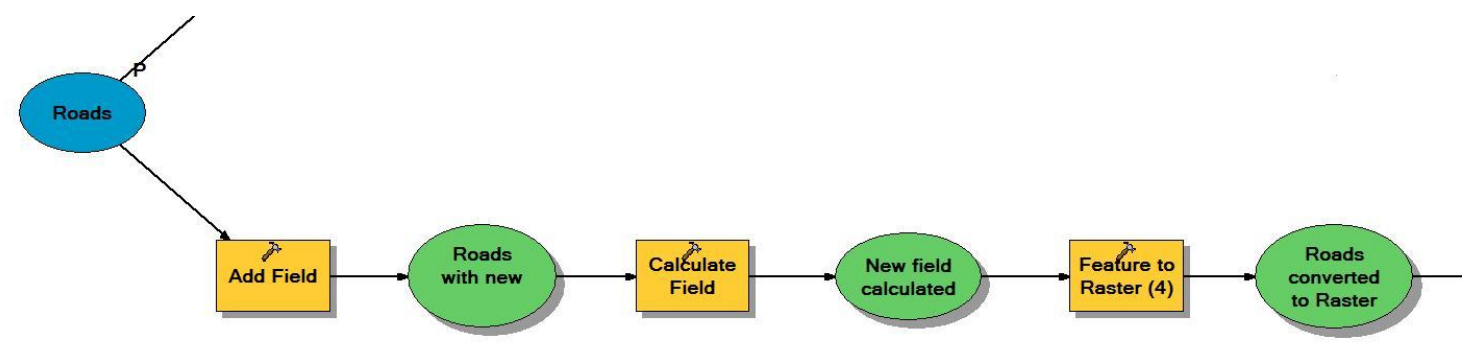

Figure 5-4. Roads Geoprocessing Analysis

\subsubsection{Waterbodies}

Water data was used to represent areas through which a vehicle could not travel. The only geoprocessing tool needed was the Feature to Raster tool. The result was a raster that contained the value one where there were water bodies and the value of no data for all other cells. Figure 5-5 shows the geoprocessing analysis for the water data.

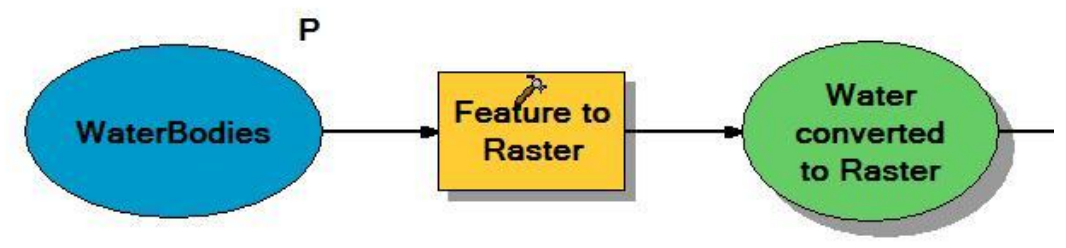

Figure 5-5. Waterbodies Groprocessing Analysis 


\subsubsection{TCP}

The traffic control points (TCP) are also known as the checkpoints. These data were used to represent areas the insurgents should avoid. The first geoprocessing tool needed was the Select tool. The Select tool was used twice, once to select the Iraqi checkpoints and once to select the US checkpoints. For each selection, the Multiple Ring Buffer tool was used to create buffers at 50,100,150, 200, 250, 300 and 1000 meter intervals. This was done because the weaponry used at checkpoints usually has an effective range of 300 meters. The 1000 meter buffer was used to represent instances where insurgents would try to not only avoid the weapon range, but also the visible range of a checkpoint. The result of this tool was vector data that had values of the intervals and no data in the rest of the extent for both of the checkpoint dataset. The next tool used was the Feature to Raster tool, which was also used on both results of the buffer. The result was two rasters that had values of 50,100,150,200,250,300, and, 1000 and no data for all other cells. The TCP geoprocessing analysis is shown in Figure 5-6.

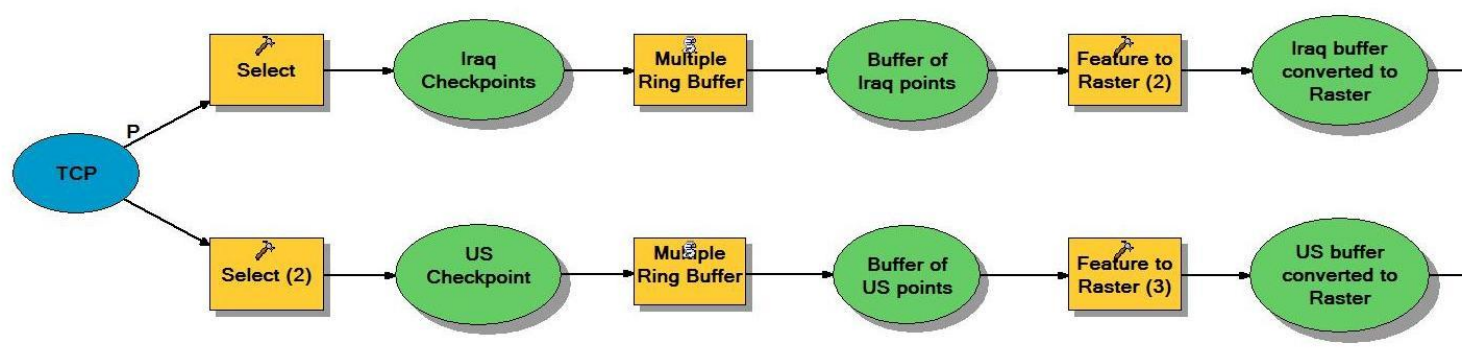

Figure 5-6. TCP Geoprocessing Analysis

\subsection{Reclassified Rasters}

The four input variables resulted in five rasters which were reclassified to calculate a cost surface for each variable. Each reclassified raster contained new values, which represented the cost to travel through each cell for each variable. Each raster was reclassified on a scale of one to nine, with the higher values representing greater costs of travel. Figure 5-7 shows all the analysis processes for all the rasters. 


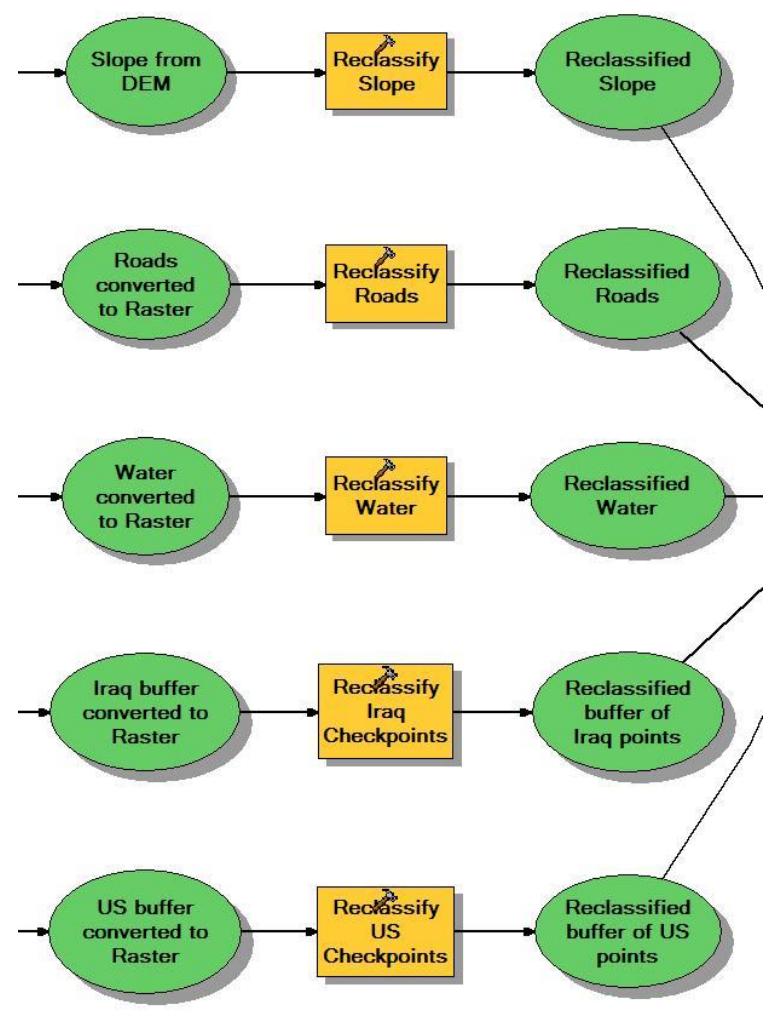

Figure 5-7. Reclassified Raster Analysis

\subsubsection{Slope}

The slope reclassification was based on the vehicle used. A HMMWV (more commonly known to civilians as a Humvee) was used as the default vehicle and the reclassifications were based on its capabilities. A HMMWV has a maximum uphill slope rating of 60 degrees and a maximum cross slope rating of 40 degrees. Since it is unknown how the least cost path is going to cross the cell, the lowest rating was used. Table 5-1 shows the slope reclassification. 
Table 5-1. Slope Reclassification

\begin{tabular}{|c|c|}
\hline Old values & New values \\
\hline $0-5$ & 1 \\
\hline $5-10$ & 2 \\
\hline $10-15$ & 3 \\
\hline $15-20$ & 4 \\
\hline $20-25$ & 5 \\
\hline $25-30$ & 6 \\
\hline $30-35$ & 7 \\
\hline $35-40$ & 9 \\
\hline $40-90$ & NoData \\
\hline NoData & NoData \\
\hline
\end{tabular}

\subsubsection{Roads}

The road classification was based on the existing road network. The raster contained values of one where the existing roads are. The new value remained the same because an existing road is the most accessible route to take. All other cells in the extent contained no data and were reclassified with the value of five. This value was chosen to make final route follow the existing roads as much as possible by making the off-road areas more costly. Table 5-2 shows the road reclassification.

Table 5-2. Road Reclassifcation

\begin{tabular}{|c|c|}
\hline Old values & New values \\
\hline 1 & 1 \\
\hline NoData & 5 \\
\hline
\end{tabular}

\subsubsection{Waterbodies}

The water data was based on water bodies considered impassable by a vehicle. The raster initially contained values of one, where the existing water bodies are. These values were reclassified to no data because they are areas through which a vehicle cannot pass. All other cells contained no data and were reclassified to one meaning passable terrain. Table 5-3 shows the classification.

Table 5-3. Waterbodies Reclassification

\begin{tabular}{|c|c|}
\hline Old values & New values \\
\hline 1 & NoData \\
\hline NoData & 1 \\
\hline
\end{tabular}

\subsubsection{TCP}

The checkpoint data resulted in two rasters: one for Iraqi (or non-US) checkpoints and the other for US checkpoints. Each raster contained the same values of 50, 100, 150, 200, 250, 300, 1000, and no data. However, they were reclassified differently because avoiding US checkpoints was more important than avoiding Iraqi checkpoints. Tables 5-4 and 5-5 show the new reclassifications for the US and Iraqi checkpoints. 
Table 5-4. US Checkpoint Reclassification

\begin{tabular}{|c|c|}
\hline Old values & New values \\
\hline 50 & 9 \\
100 & 8 \\
\hline 150 & 7 \\
200 & 6 \\
\hline 250 & 5 \\
300 & 4 \\
1000 & 2 \\
\hline NoData & 1 \\
\hline
\end{tabular}

Table 5-5. Iraqi (non-US) Checkpoint Reclassification

\begin{tabular}{|c|c|}
\hline Old values & New values \\
\hline 50 & 8 \\
\hline 100 & 7 \\
150 & 6 \\
\hline 200 & 5 \\
250 & 4 \\
\hline 300 & 3 \\
\hline 1000 & 2 \\
\hline NoData & 1 \\
\hline
\end{tabular}

\subsection{Weighted Sum}

After all five rasters were reclassified, a cost surface was produced (see Figure 58). The Weighted Sum tool was the only geoprocessing tool needed to create that surface. This tool combines multiple rasters and creates an integrated analysis. This tool is very similar to the weighted overlay tool, but it allows for floating-point values instead of just integers. In the future the reclassification can be improved to a continuous scale. 


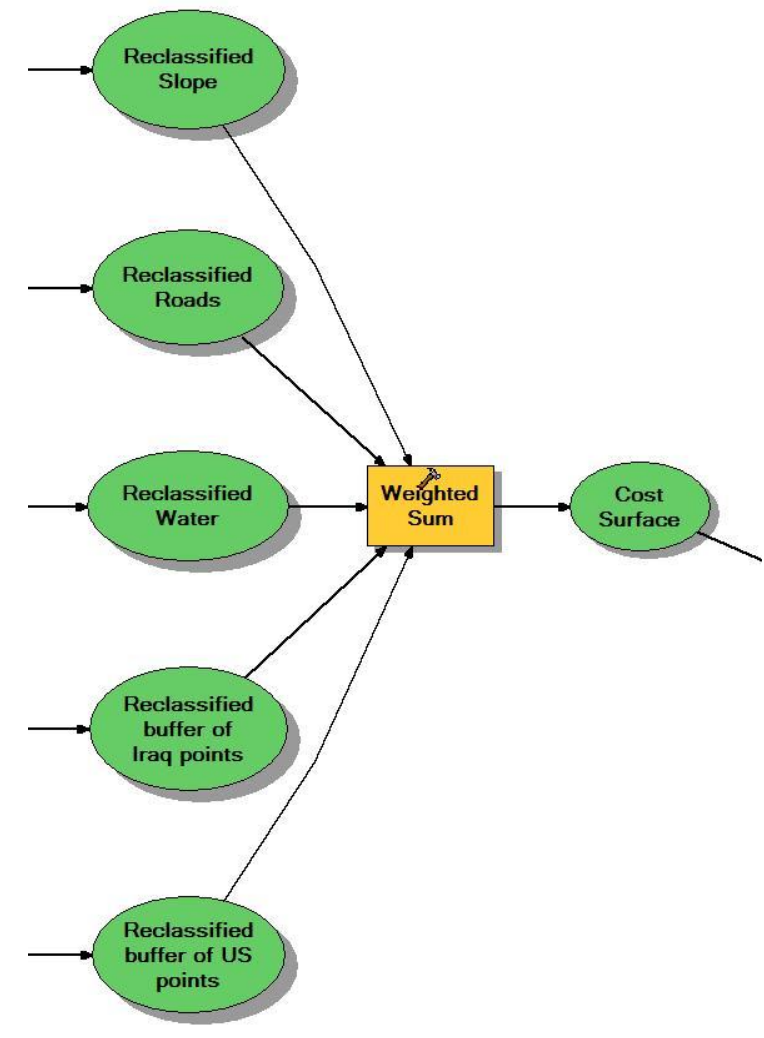

Figure 5-8. Weighted Sum Tool

The Weighted Sum tool used the five reclassified rasters as inputs. The tool also requires a value in the weight field for each raster. By default, all weights are set to one (see Figure 5-9).

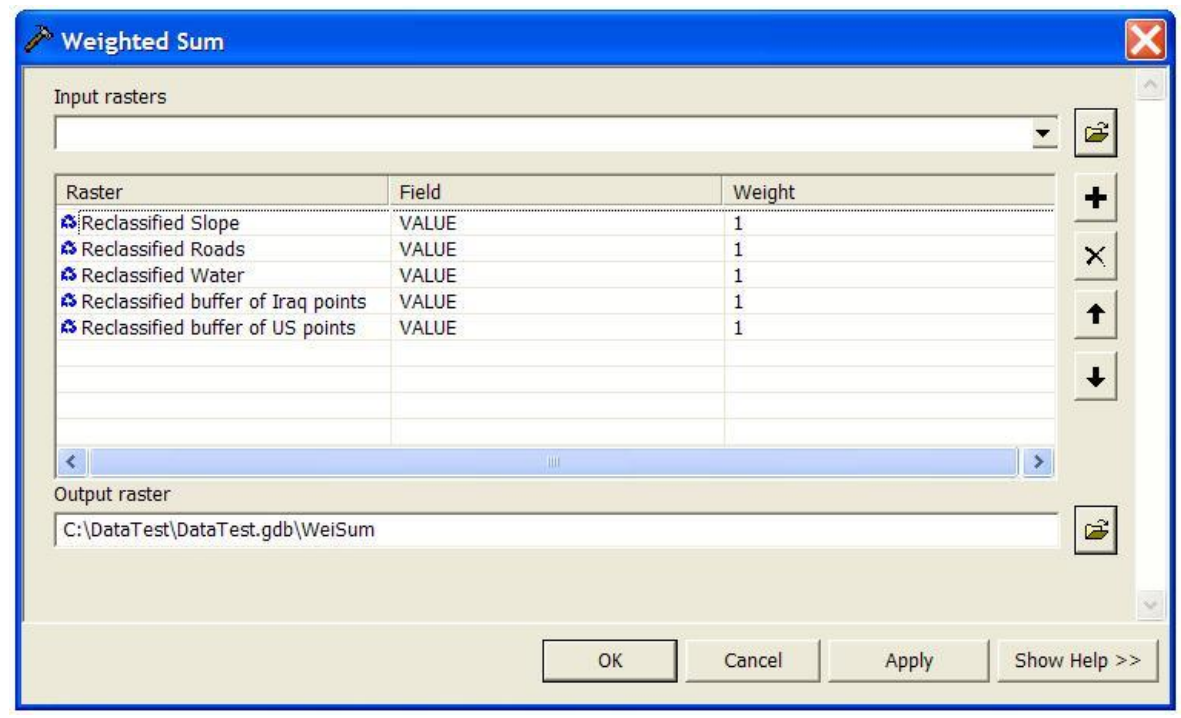

Figure 5-9. Weight Sum Tool Interface 


\subsection{Least Cost Path}

The Cost Distance and Cost Path tools were used to generate the least cost path. The Cost Distance tool calculated "the least accumulative cost distance for each cell to the nearest source over a cost surface" (ESRI, 2008). The Cost Distance tool required two inputs: a cost surface raster and a start point. The cost surface was the raster produced by the Weighted Sum tool. One of the MOUT site points was used as the start point. The Cost Distance tool produced a cost distance raster and a backlink raster. The cost distance raster identified, "for each cell, the least accumulative cost distance over a cost surface to the identified source locations" (ESRI, 2008). The backlink raster contained "values of 0 through 8 , which define the direction or identify the next neighboring cell (the succeeding cell) along the least accumulative cost path from a cell to reach its least cost course" (ESRI, 2008).

The Cost Path tool had three inputs: the cost distance raster, the backlink raster that the cost distance tool generated, and the destination end point. Another MOUT site point was used as the end point. The Cost Path tool calculated the least cost path from the start point to the end point and created a cost path raster. This least cost path represented a plausible off-road route that an insurgent might take to avoid detection. Figure 5-10 shows the geoprocessing for the least cost path analysis.

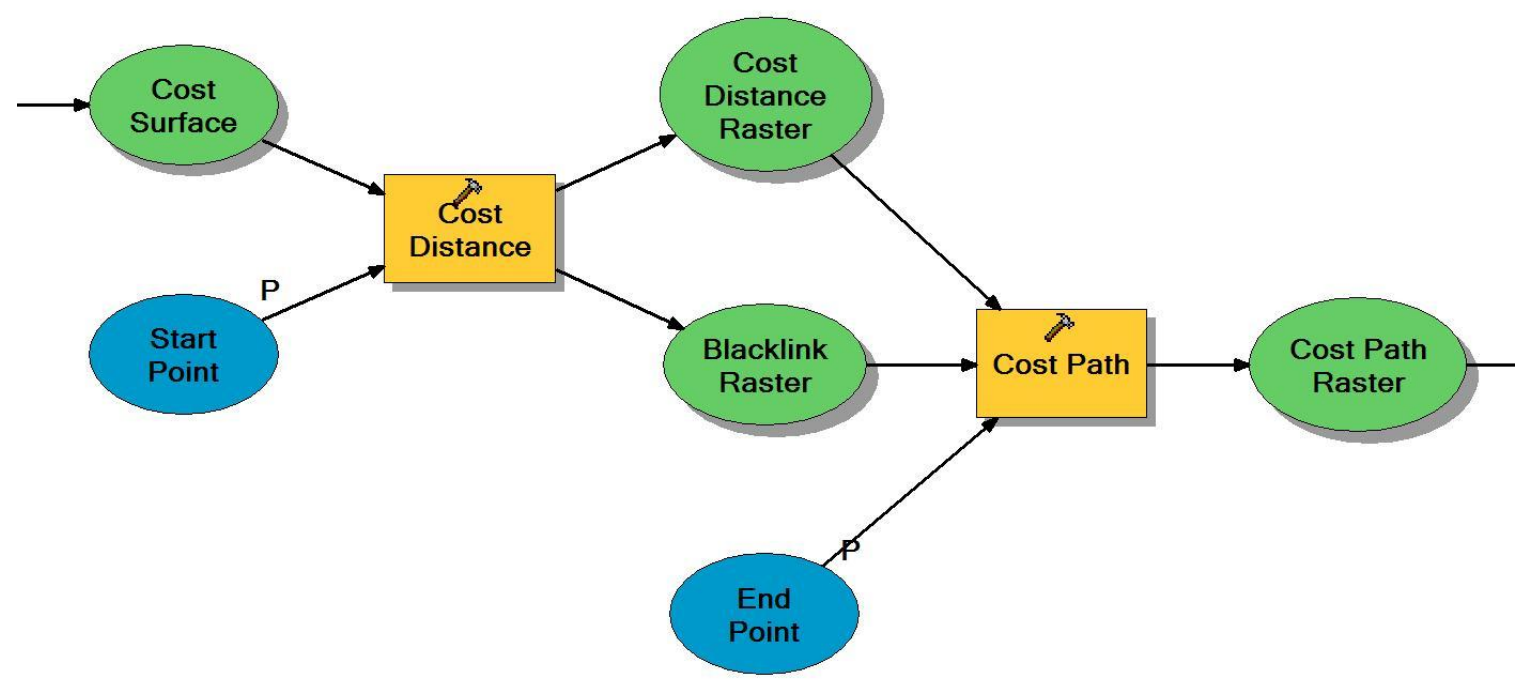

Figure 5-10. Least Cost Path Analysis

\subsection{Software Compatibility}

The final steps of the geoprocessing analysis included making the least cost path compatible with VBS2 software. The final goal of these steps was to generate the final route as vector data with added attributes.

The Raster to Polyline tool was used to convert the cost path raster into vector format as a linear feature. Once in vector format, the Add Field tool was used to add a 
field name Type. The Calculate Field tool was then used to calculate the type field with "Off-road" as the attribute. This field was added to distinguish the new path as an offroad path and not a path using only the existing roads. Another field was added and calculated which used the same two tools. A field, Speed, was added to establish a speed limit (in miles per hour) for the vehicle when it is on the off-road section of the path. The Merge tool was the final tool used. This tool merged the existing road data and the new off-road path to produce a new feature dataclass. The software compatibility process can be seen in Figure 5-11.

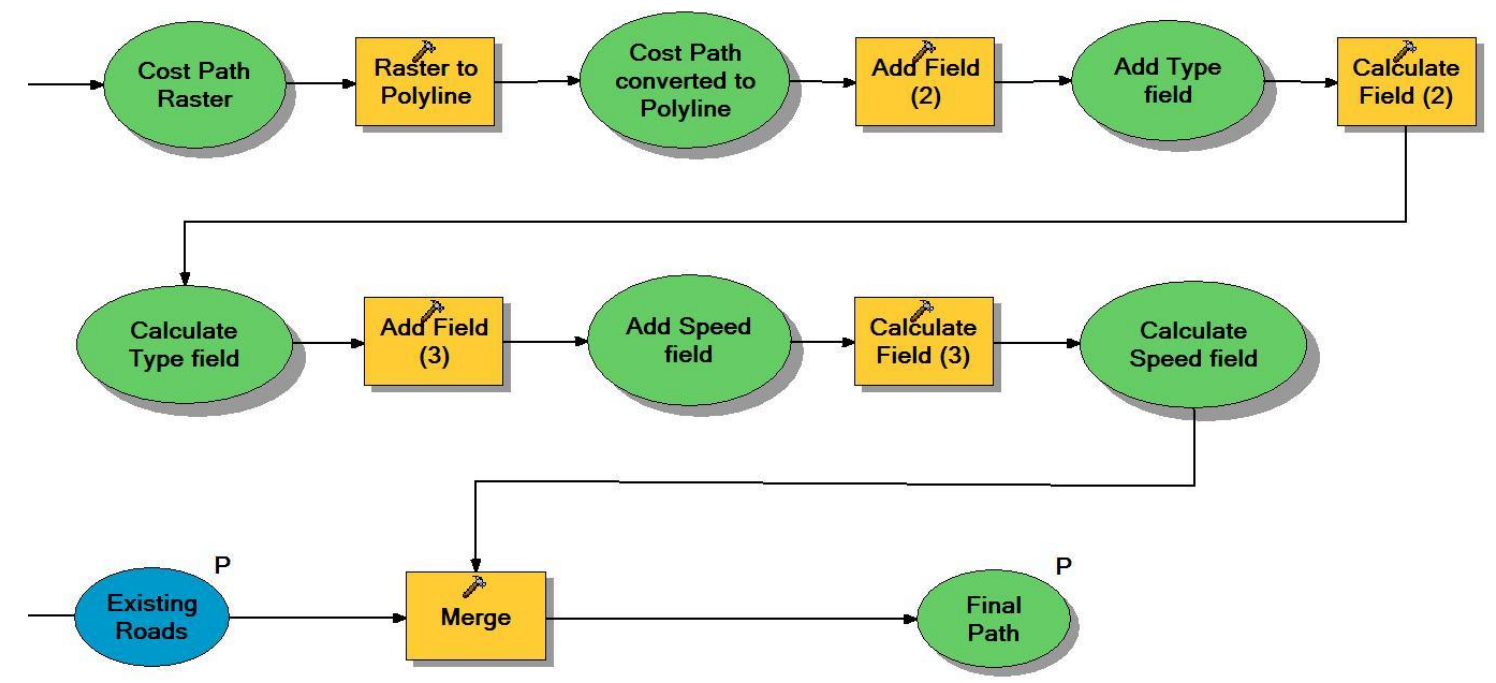

Figure 5-11. Software Compatibility Analysis 


\section{Model Development}

Once the model was completed, the next task was to develop the model tool. The model was further developed to test the functionality of the tool. Many trial and error tests were completed, but this section only covers the significant results. The analyses focused on changing the start and end points. The generated routes displayed in this chapter did not go through the post-model tasks.

\subsection{Generating Routes from a Single Point}

This section covers tests that contained only one start point and only one end point. The first test used all the default settings for each tool and had point A as the start point and point $\mathrm{B}$ as the end point. The results of this test followed the existing road (see Figure 6-1). This did not illustrate any deviations from the existing roads to avoid the checkpoints, so additional tests were necessary.

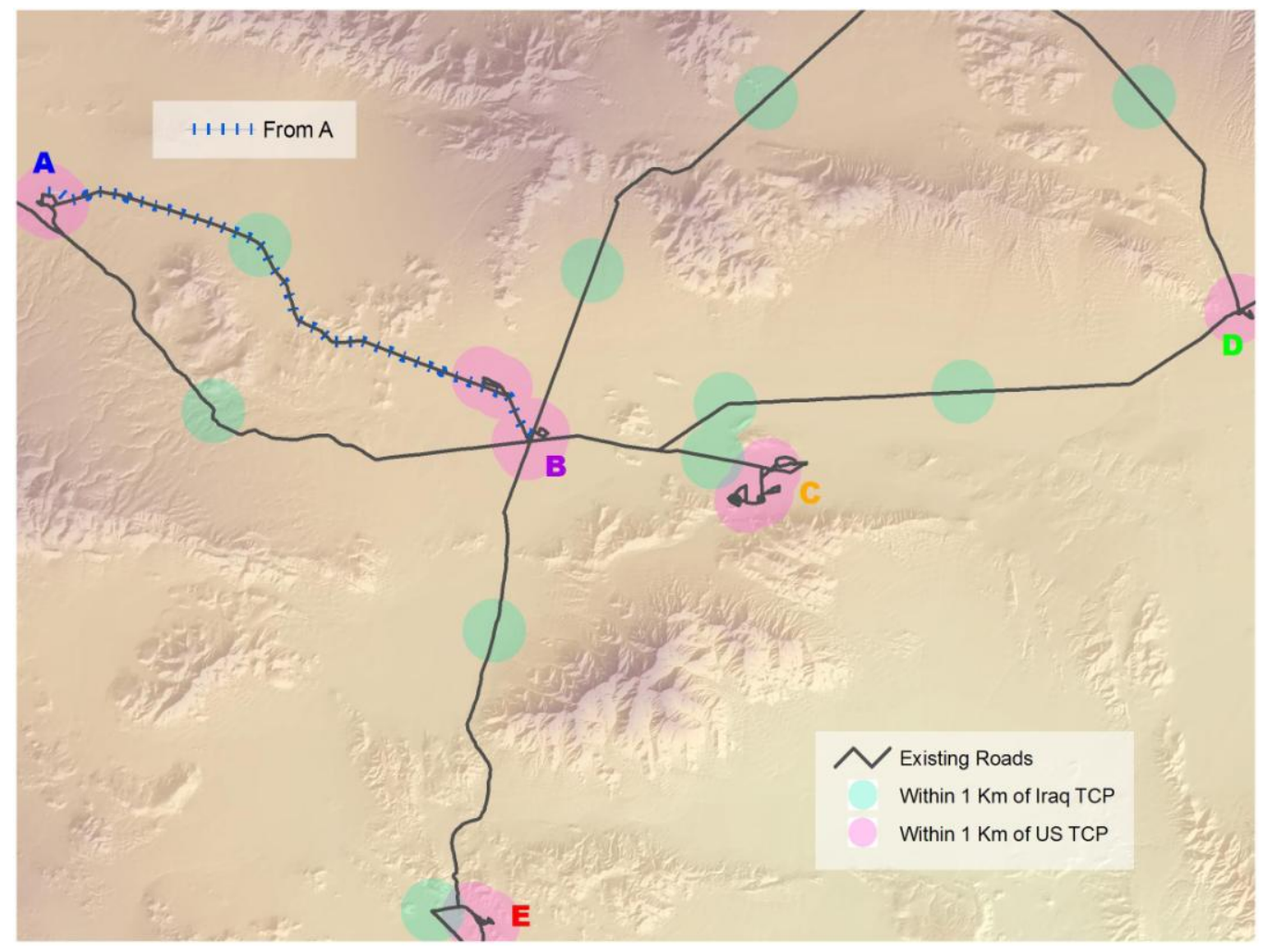

Figure 6-1. First Test from One Point to One End Point

A second test was conducted that increased the weights for both the Iraqi (nonUS) and US checkpoints from the default of one to five, using the same start and end points. The results of this test (see Figure 6-2) demonstrated that the new route avoided the checkpoints, identifying that the model correctly avoided checkpoints if the weights were set sufficiently higher. 


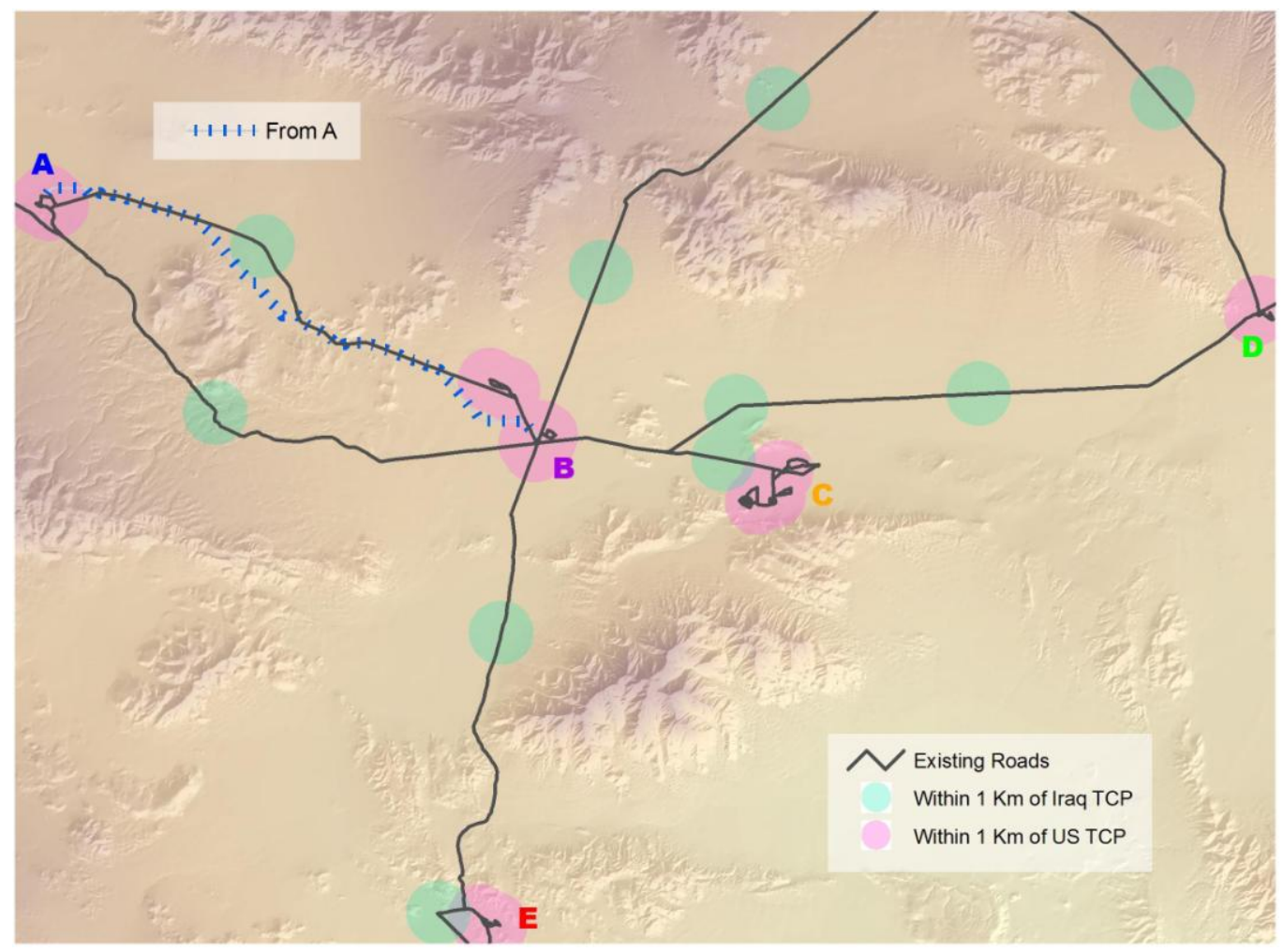

Figure 6-2. First Test That Avoided Checkpoints

\subsection{Results with Multiple End Points}

More trials were conducted to test the capabilities of the model. To generate multiple paths, multiple end points were used. The next test used point $\mathrm{A}$ as the start point and points $\mathrm{B}, \mathrm{C}, \mathrm{D}$, and $\mathrm{E}$ as the end points. The weights for both the Iraqi (non-US) and US checkpoints were set to eight with the remaining weights set to one. The results of this test (see Figure 6-3) demonstrated that the model was able to generate four separate routes, each route to one of the end points. 


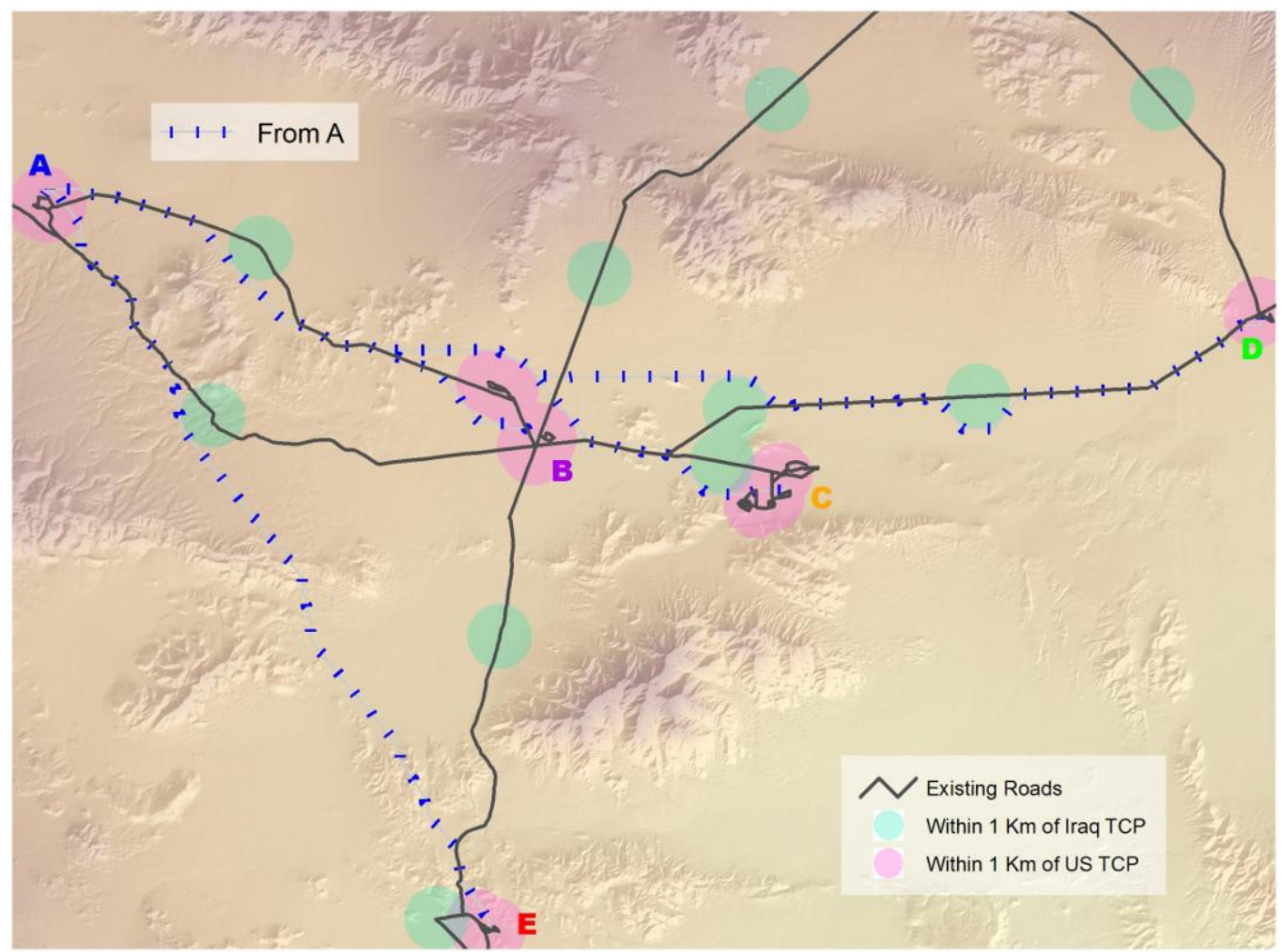

Figure 6-3. Test From One Point to Multiple End Points

\subsection{Generating Routes from Multiple Start Points}

Tests that included multiple start points were conducted to test the capabilities of the model as well. The checkpoints weight value was set at a value of eight for all tests in this section.

\subsubsection{Results with a Single End Point}

The test conducted for this situation contained points B, C, D and E as the start points and point $A$ as the end point. The output included only a single path from point $B$ to point A, but this method did not work as expected (see Figure 6-4). Although it was expected that the model would generate separate routes from each of the four start points, it in fact identified only the shortest route of all possible routes from B, C, D, or E to A. 


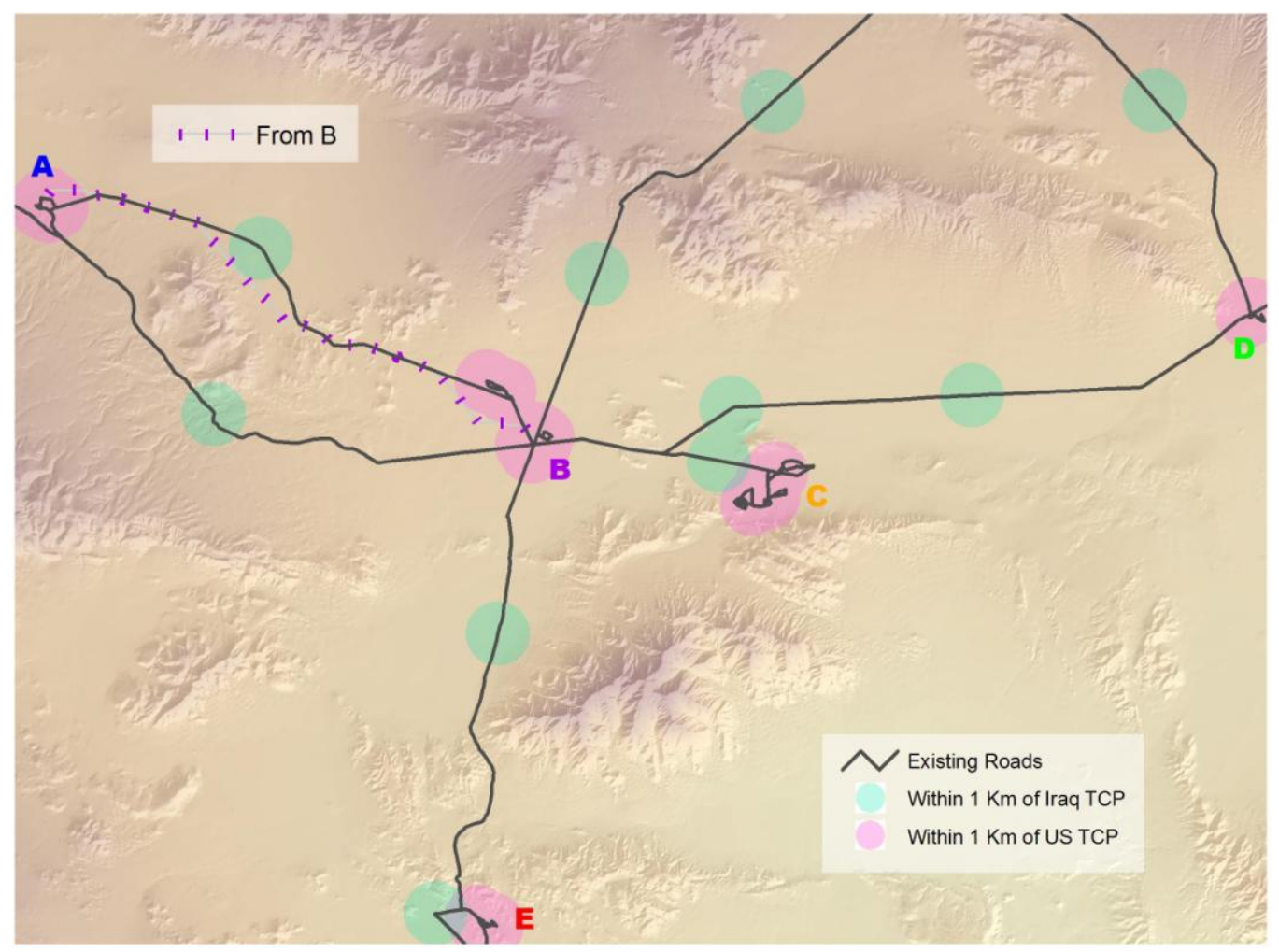

Figure 6-4. Test with Multiple Points and One End Point

\subsubsection{Results with Multiple End Points}

The first test conducted for this situation contained the points A and B as the start points and points $C, D$, and $E$ as end points. The result included only three paths, from $B$ to C, B to D, and B to E (see Figure 6-5). Apparently the tool identified the least cost paths from point $A$ or $B$, to $C, D$ and $E$. Since all of the routes from point A were more costly than the paths from point $\mathrm{B}$, only those from point $\mathrm{B}$ were identified. 


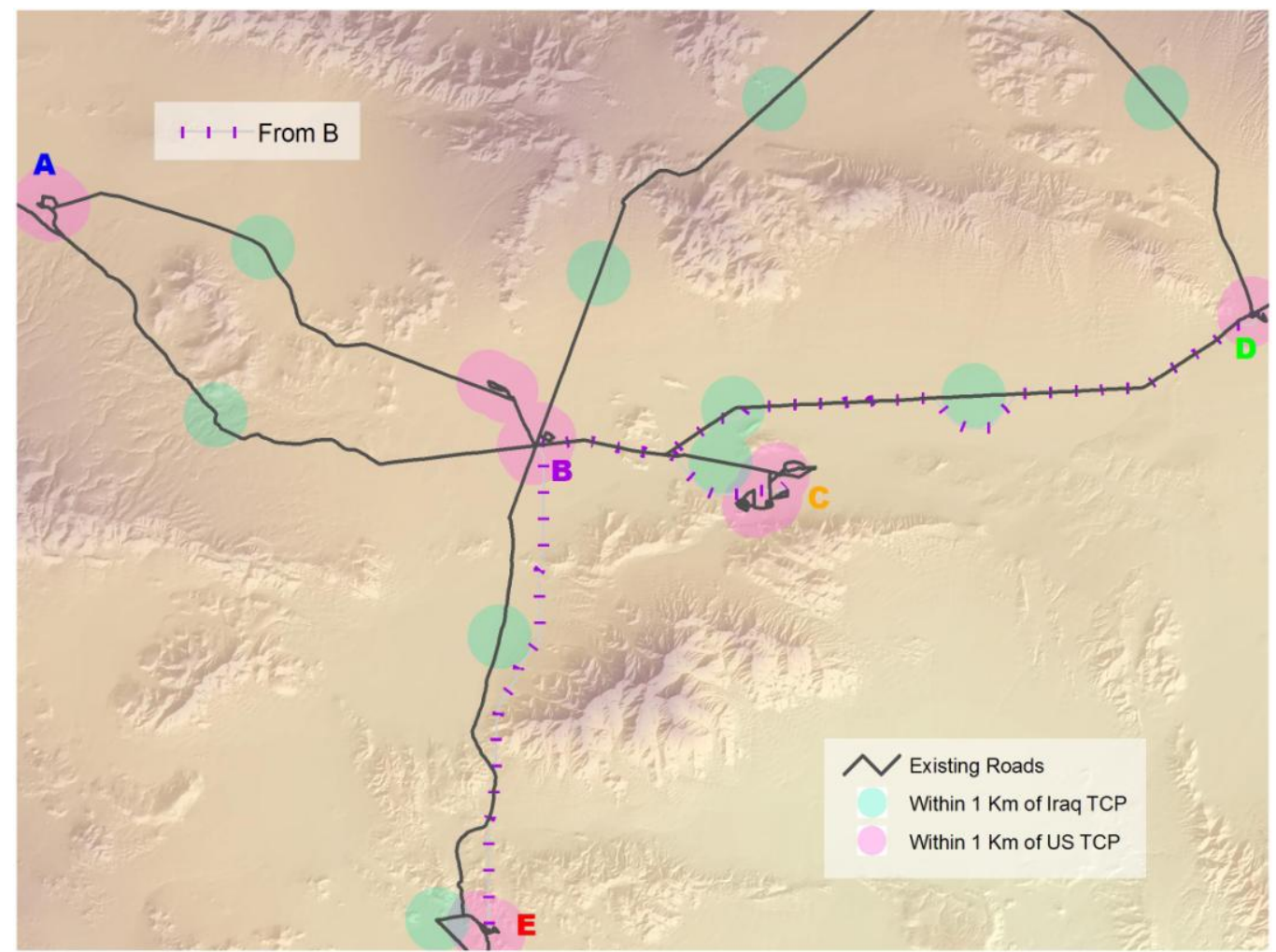

Figure 6-5. Multiple Start and End Points Test

A second test was conducted with points A and D as the start points and points B, $\mathrm{C}$, and $\mathrm{E}$ as the end points. The results supported the assumption. The model again identified three routes to the three destinations, but this time two of the routes began at point $\mathrm{A}$ and one began at point D (see Figure 6-6). 


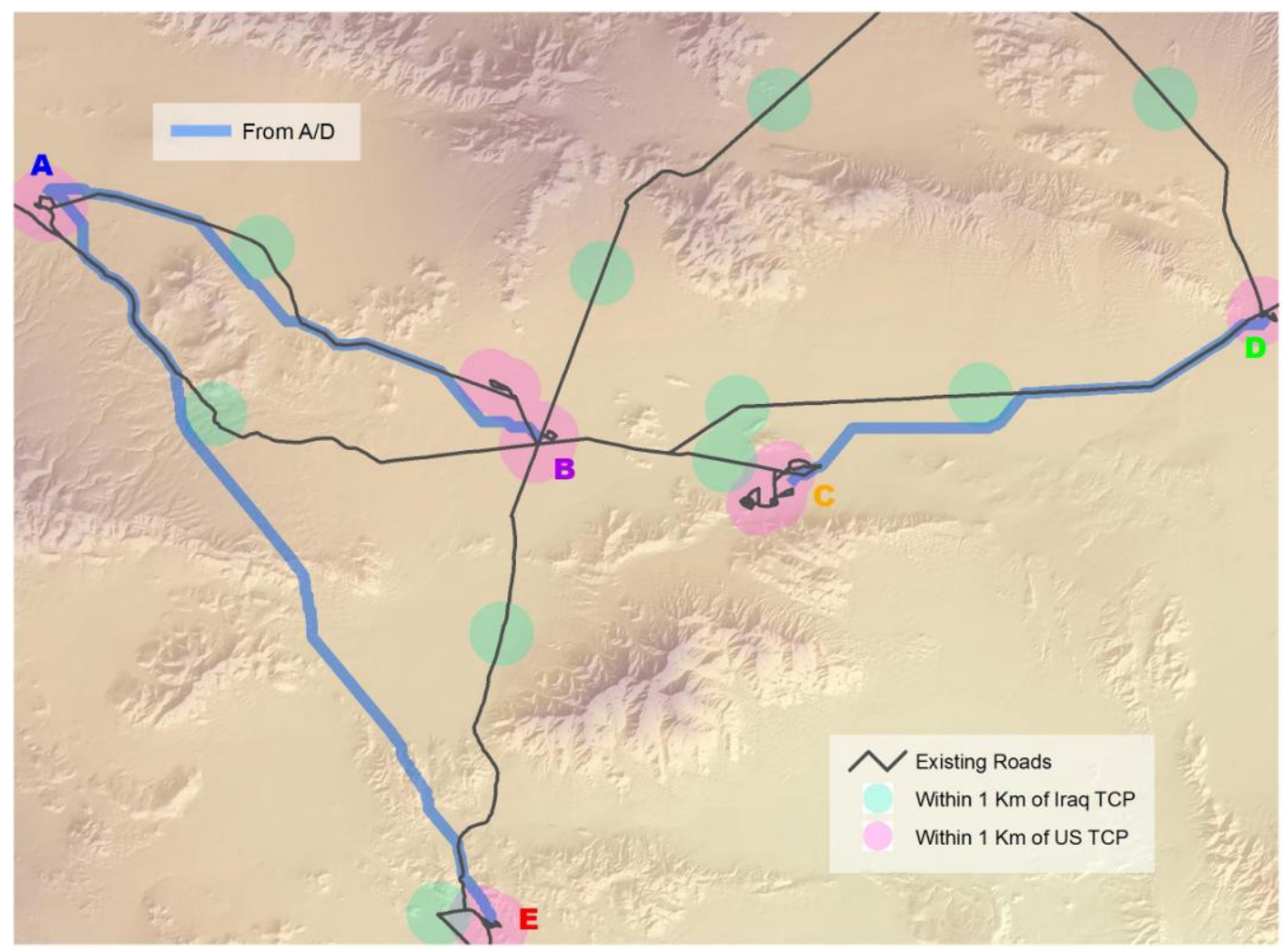

Figure 6-6. Different Multiple Start and End Points Test

\subsubsection{Results with the Same End Points}

Using the same start and end points was tested in an attempt to generate all possible routes between the five MOUT sites. Previously, the model tool was run five different times with different inputs for the start and end points. However, using the same start and end points failed and routes were not generated.

\subsection{Discussion on Limitations}

To generate as many routes as possible at one time, it is recommended that the model have one start point and have multiple end points. However, using multiple start points and end points can be useful in another type of analysis. A situation could involve a site needing more resources and this analysis would help determine which other site would be the most efficient to provide those resources (given other factors being equal). However, there may be other factors to include in allocation problems such as transportation costs, supplies and demand. 


\section{7. $\quad$ Model Testing}

This chapter focuses on the model's ability on changing the weights, changing the resolution of the rasters, and using different spatial data. The purpose of conducting these tests was to demonstrate that the model tool is more than a prototype by generate routes for different scenarios and has the ability to be used on any dataset.

\subsection{Changing the Weights of the Data Layers}

This section focuses on changing the weights for the Iraqi checkpoints, US checkpoints, and slope layers to generate routes for different scenarios.

\subsubsection{Checkpoints}

The first series of tests involved changing the Iraqi and US checkpoint weights. When the Iraqi and US checkpoints each had weights of three, the new route avoided the checkpoints by at least 300 meters as shown in Figure 7-1. All possible routes between the five points that have the Iraqi and US checkpoint weights at three are also shown. Although many of the routes appear to go directly through the checkpoint, they in fact detour around the checkpoint by at least 300 meters, except for the route from point B to point E.

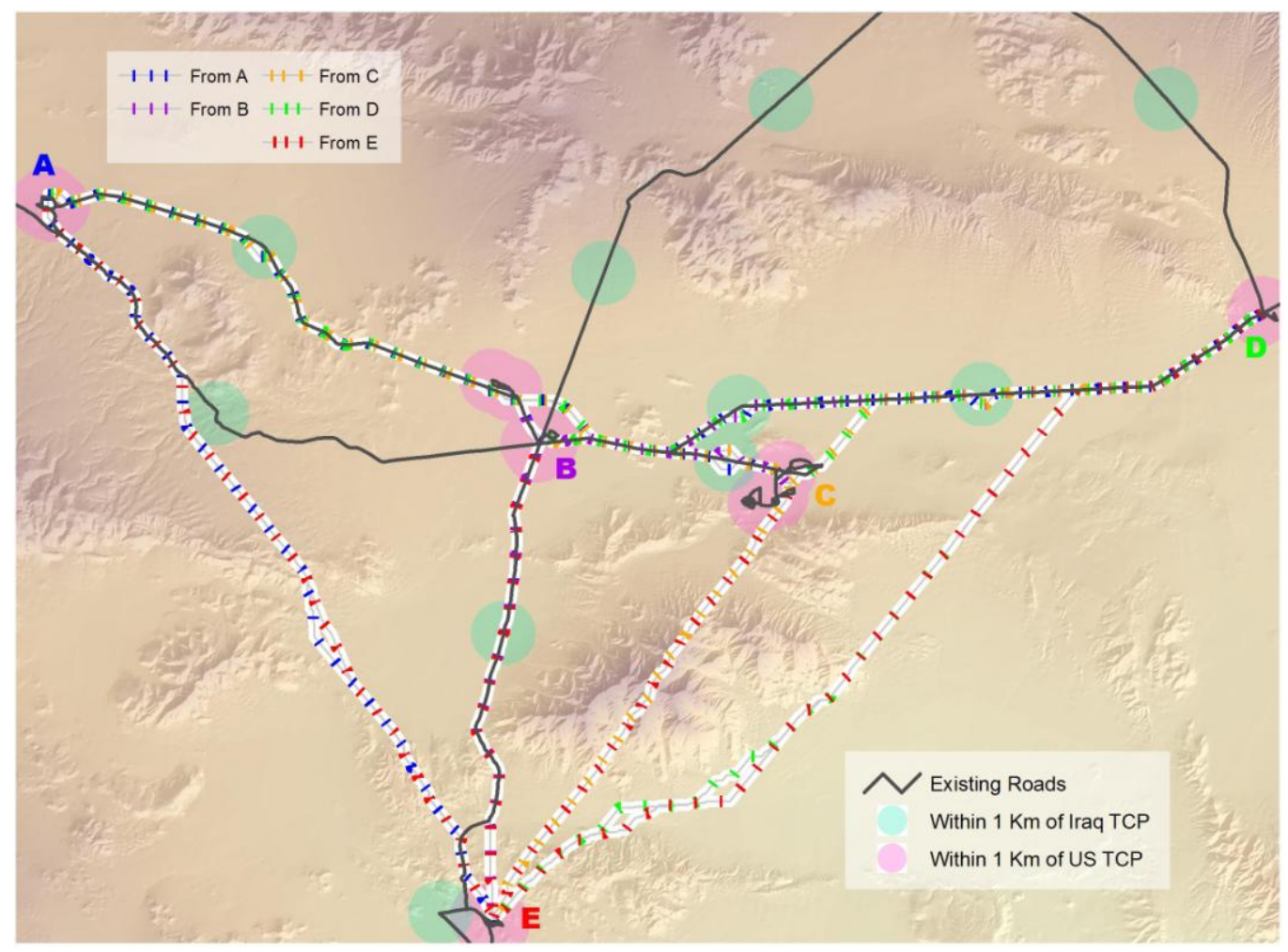

Figure 7-1. All Routes Between MOUT Sites that Avoid Checkpoints at 300 Meters 
Figure 7-2 shows the results when the weights for both checkpoints were raised from three to eight. As in the previous test, point A was the starting point and points B, C, $\mathrm{D}$, and $\mathrm{E}$ were the end points. Figure 7-2 also shows all possible routes between the five points that have the Iraqi and US checkpoints weights at eight. In virtually all cases, the increased weights yield routes that avoided the checkpoints by at least 1000 meters. In one instance, the route only avoided an Iraqi checkpoint by 300 meters. This map also illustrates that routes between the same two points do not always follow the same path, as shown between points $\mathrm{A}$ and $\mathrm{E}, \mathrm{C}$ and $\mathrm{D}$, and $\mathrm{D}$ and $\mathrm{E}$. These differing routes are the result of the processes used to determine the least cost path. The process is sensitive to the direction of travel when a cell is entered from which there are two or more equally low cost exit paths.

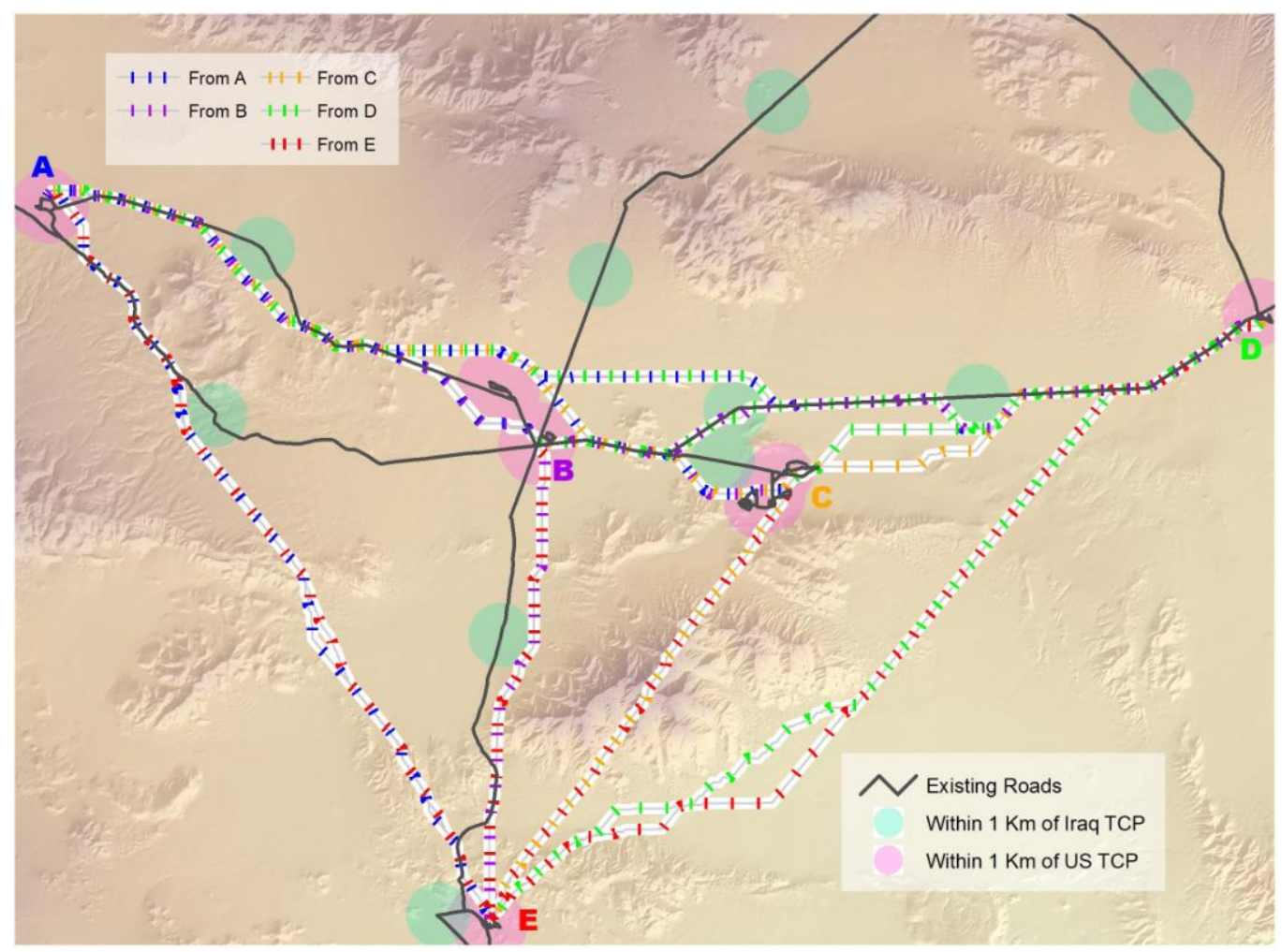

Figure 7-2. All Routes Between MOUT Sites that Avoid Checkpoints at 1000 Meters

The next two scenarios examined the effects of using different weights for the different types of checkpoints (US vs. Iraqi). The first scenario gave the Iraqi checkpoints a weight of four and the US checkpoints a weight of seven. The model generated routes that avoided the Iraqi checkpoints by at least 300 meters and avoided the US checkpoints by at least 1000 meters (see Figure 7-3). This scenario was tested using only point A as the start point and points $\mathrm{B}, \mathrm{C}, \mathrm{D}$, and $\mathrm{E}$ as the end points. 


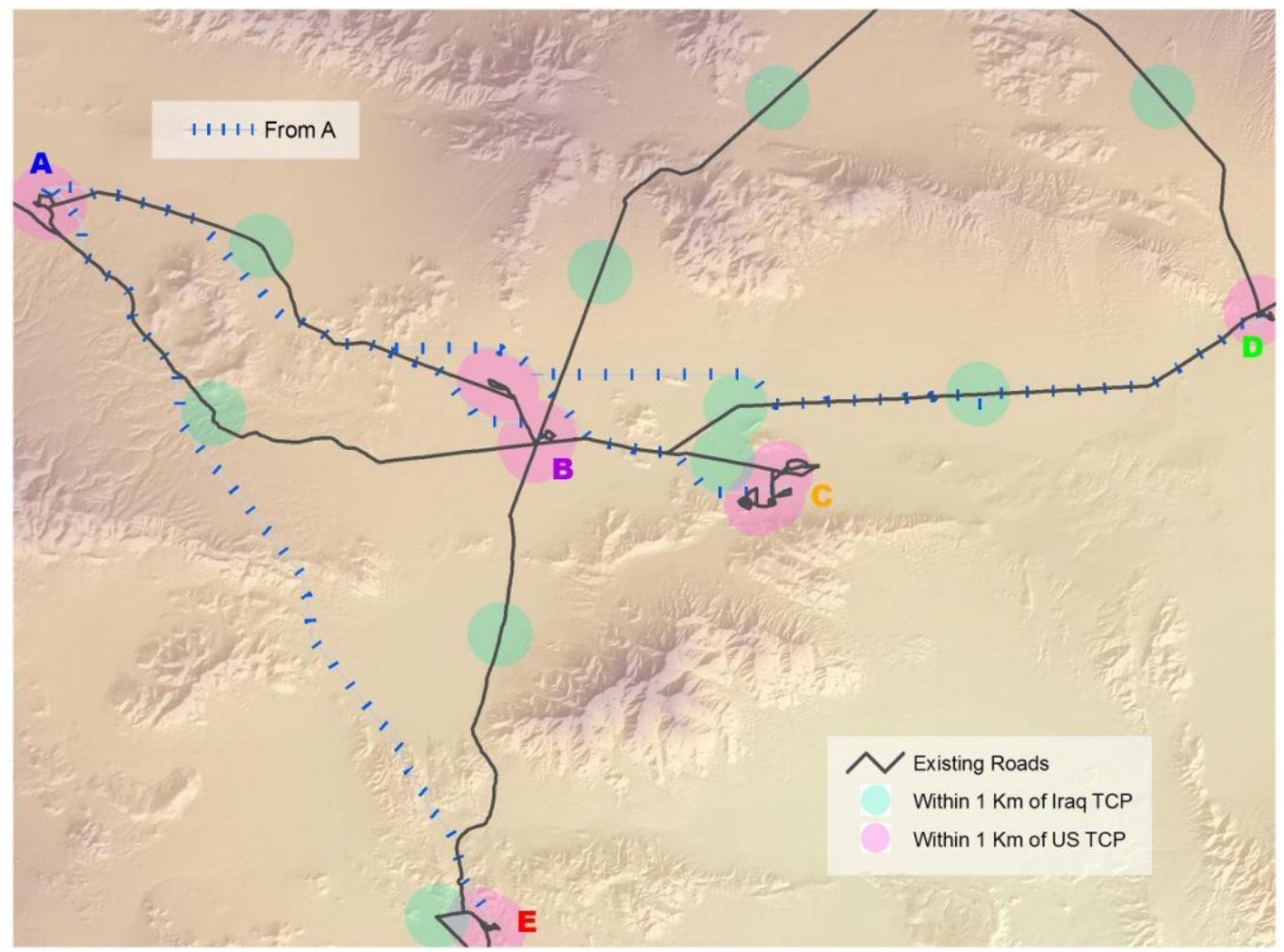

Figure 7-3. Routes Avoiding US TCPs at a Greater Distance than Iraqi TCPs

The next scenario gave the Iraqi checkpoint a weight of eight and the US checkpoint a weight of two, and generated routes that avoided the Iraqi checkpoints by at least 1000 meters and the US checkpoints by at least 300 meters (see Figure 7-4). 


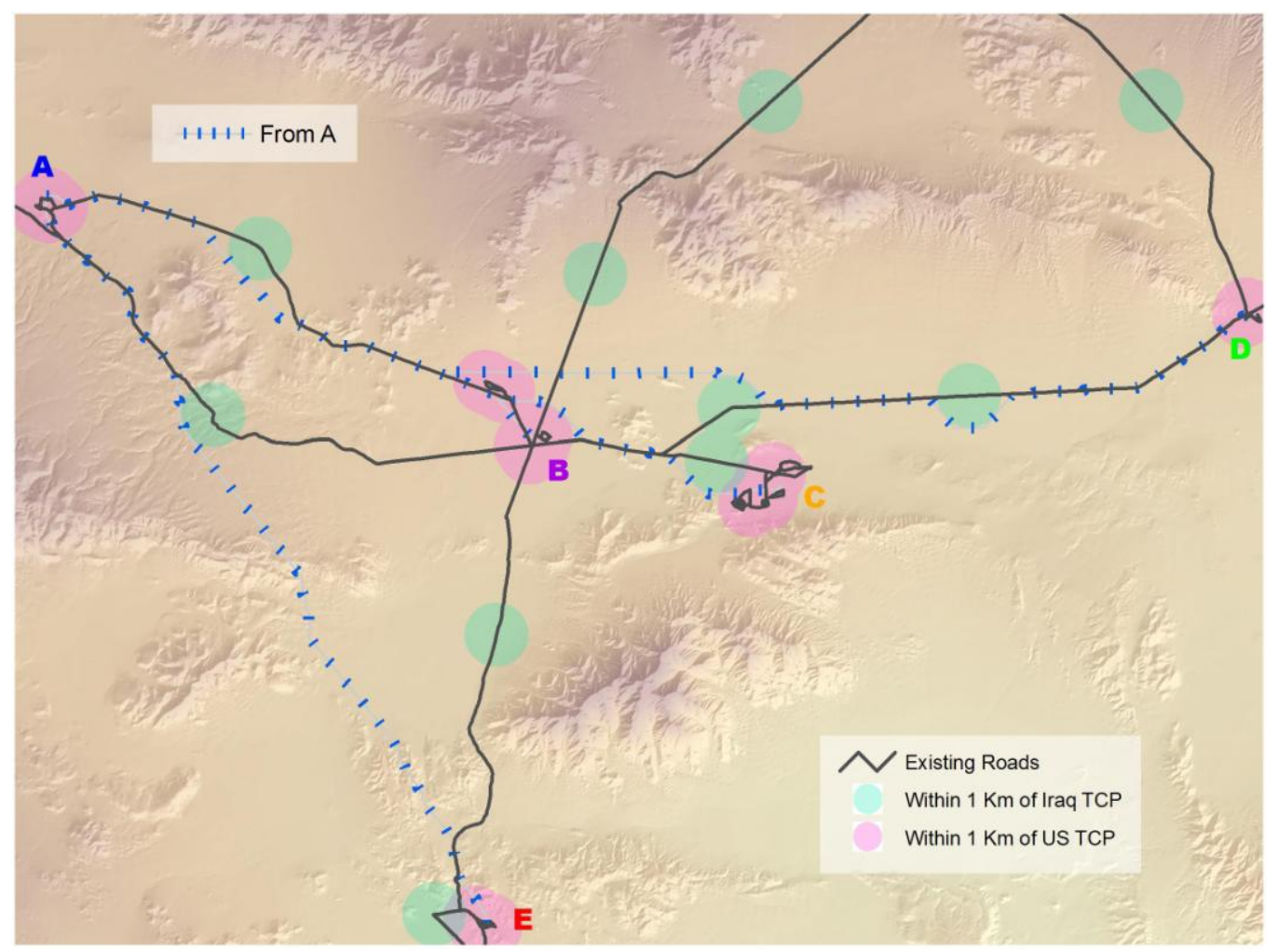

Figure 7-4. Routes Avoiding Iraqi TCPs at a Greater Distance than US TCPs

\subsubsection{Changing the Slope Weight}

The next situation tested what would happen if the weight of the slope layer was changed. Several tests were conducted using point A as the start point and points B, C, D, and $\mathrm{E}$ as the end points. The checkpoints weights remained unchanged at eight, and model was tested with several values for the slope layer over the range from one to 20 . Each result was similar in that the routes avoided the checkpoints at 1000 meters but did not go back to the road. The paths did, however, change by continuing through areas with a lower slope value instead of following the road. After analyzing the area, the results were as to be expected considering the terrain between those points.

Changing the slope weight was tested again with a new set of points. Point $\mathrm{E}$ was used as the start point and points A, B, C, and D were used as the end points. The value of the slope weight was set to eight. Changing the slope weight on this set of points had an effect on the paths. The path to point B stayed more on the road and the path to point $\mathrm{C}$ avoided the mountains. Figure 7-5 shows the difference between the paths when the slope value was changed. 


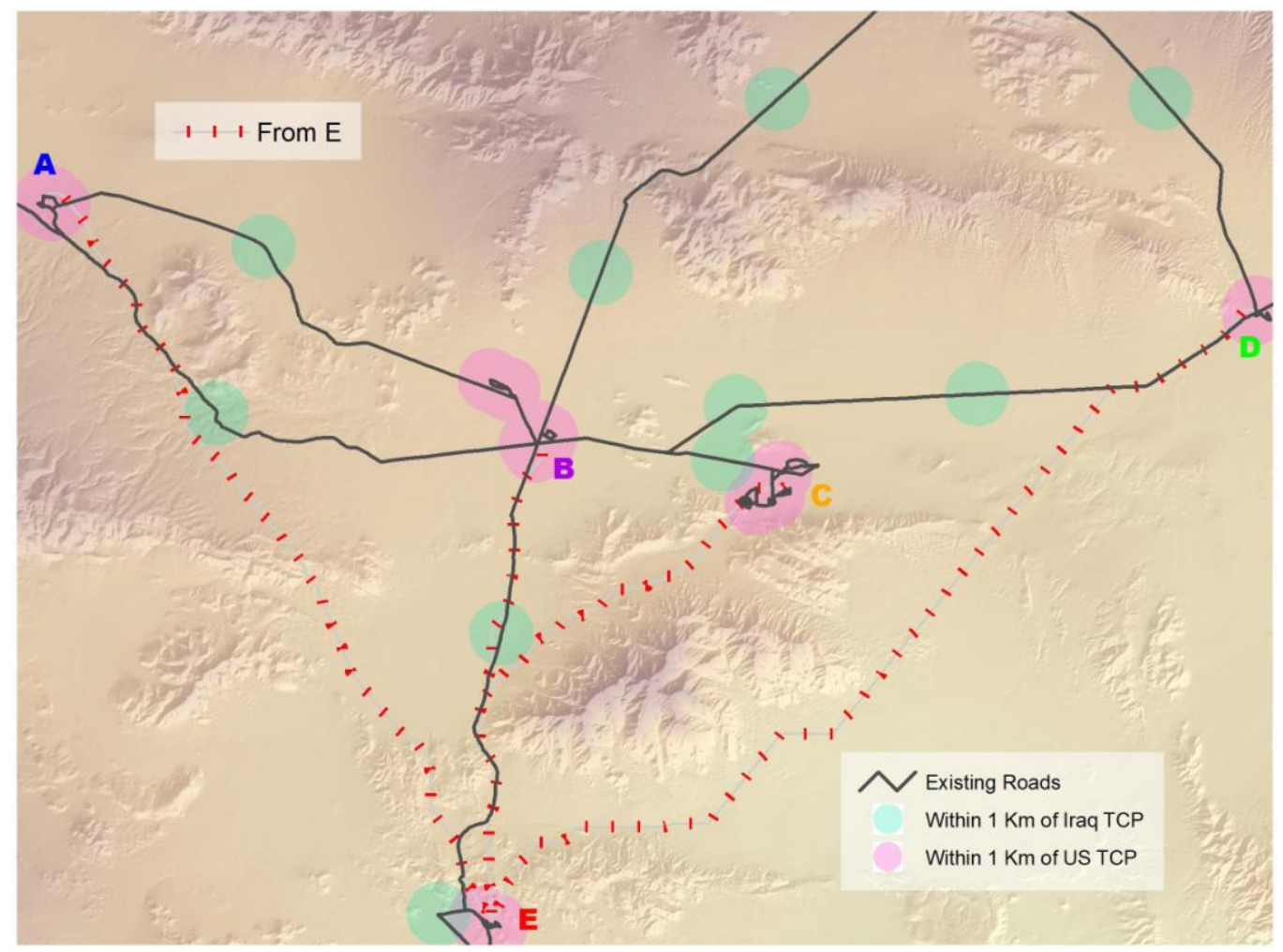

Figure 7-5. Test with a Different Slope Weight

\subsection{Changing the Resolution of the Vector-based Datasets}

Until this point in the model development and testing, all analyses were carried out with 10-meter resolution datasets, to achieve realistic, detailed results. The client anticipated applying the model in other geographic areas where 10-meter resolution elevation data might not be available. In preparation for this, the model was tested with courser resolution versions of the vector based data. This section, describes the results of testing 30-, 100-, and 1000-meter resolution representations of the vector data, with the high resolution 10-meter elevation data. This testing resulted after first attempting to use the client provided DEMs.

\subsubsection{Using Client's DTED Format DEMS}

The first test involved different DEM inputs to the model. The client provided 30 , 100-, and 1000-meter resolution of the DEM. All three elevation datasets were tested and did not work. The elevation datasets were in a digital terrain elevation data (DTED) format. This format contained angular measurements for the longitude and latitude and elevation in meters. Since all three measurements were not the in the same units, the Slope tool failed and the model did not work. The client stated that other sources for a DEM that are compatible with this model tool will be used and the model does not have to work with the DTED format. 


\subsubsection{Using Coarser Resoultion Raster Versions of Vector Data}

Four vector-based datasets (roads, waterbodies, US checkpoints, and Iraqi checkpoints) were converted to raster datasets using the Feature to Raster tool. In all previous tests, the cell size used with that tool was equal to the size of the DEM cell size, that is, .000093 angular degrees (or approximately ten meters). All previous tests were conducted with this cell size. The client also provided three DEM's of this area of different resolutions. Three tests were conducted with these three cell sizes to show how cell size affects the results of the model. For each test, point A was used as the start point and points B, C, D, and E were used as the end points. The slope, road, and waterbodies weights were set to one, while both checkpoints weights were at eight. Figures 7-6 through 7-8 show the three cell sizes (from smallest to largest) and the results. As the cell size increased, the paths became coarser. The paths were essentially identical, but smaller cell sizes yielded smoother paths. The only significant difference in the three sets of paths appears in Figure 7-8, where the coarse rasters allowed the path to not follow the roads at all.

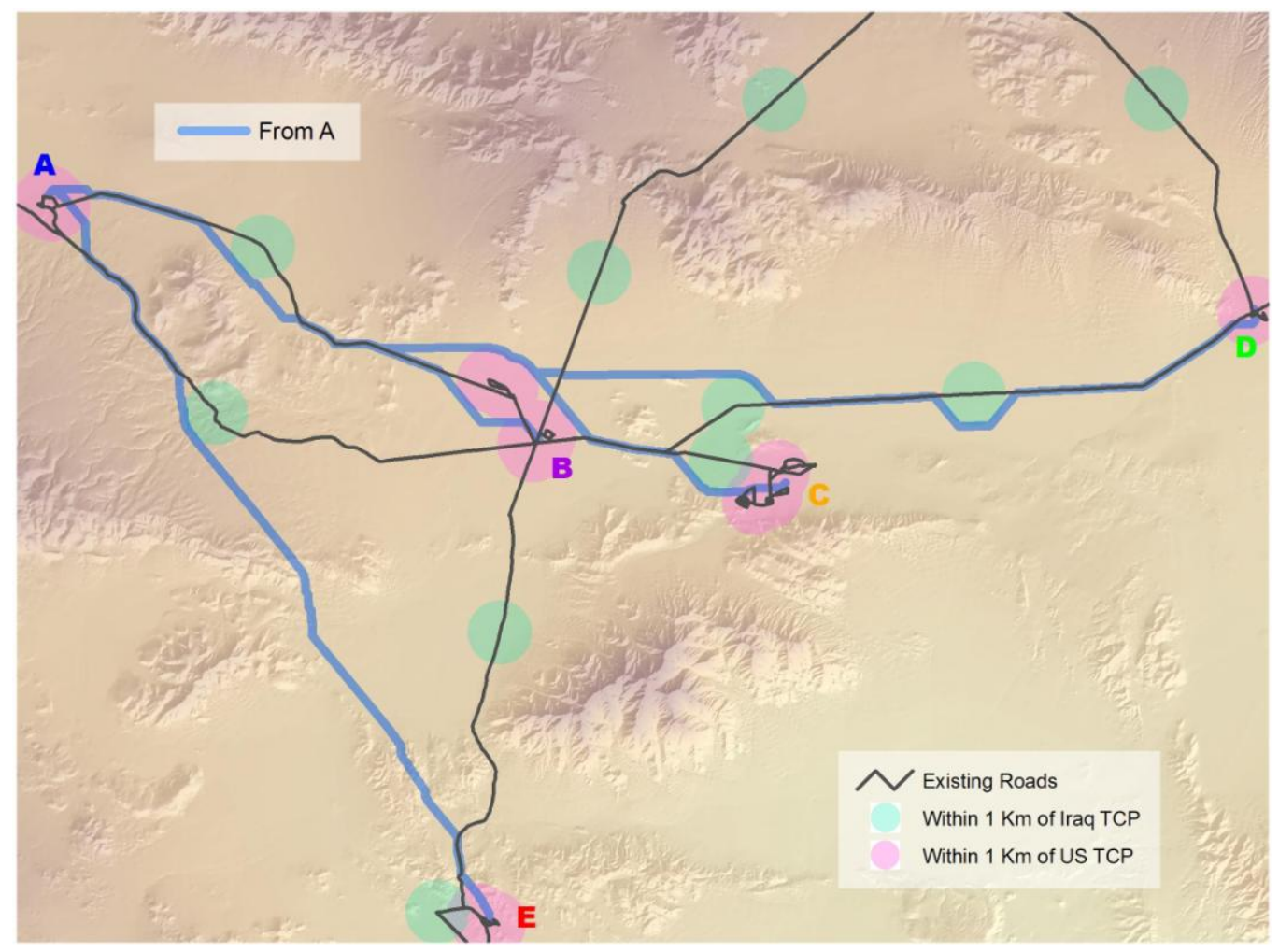

Figure 7-6. Vector-based Layers at 30-meter Resolution 


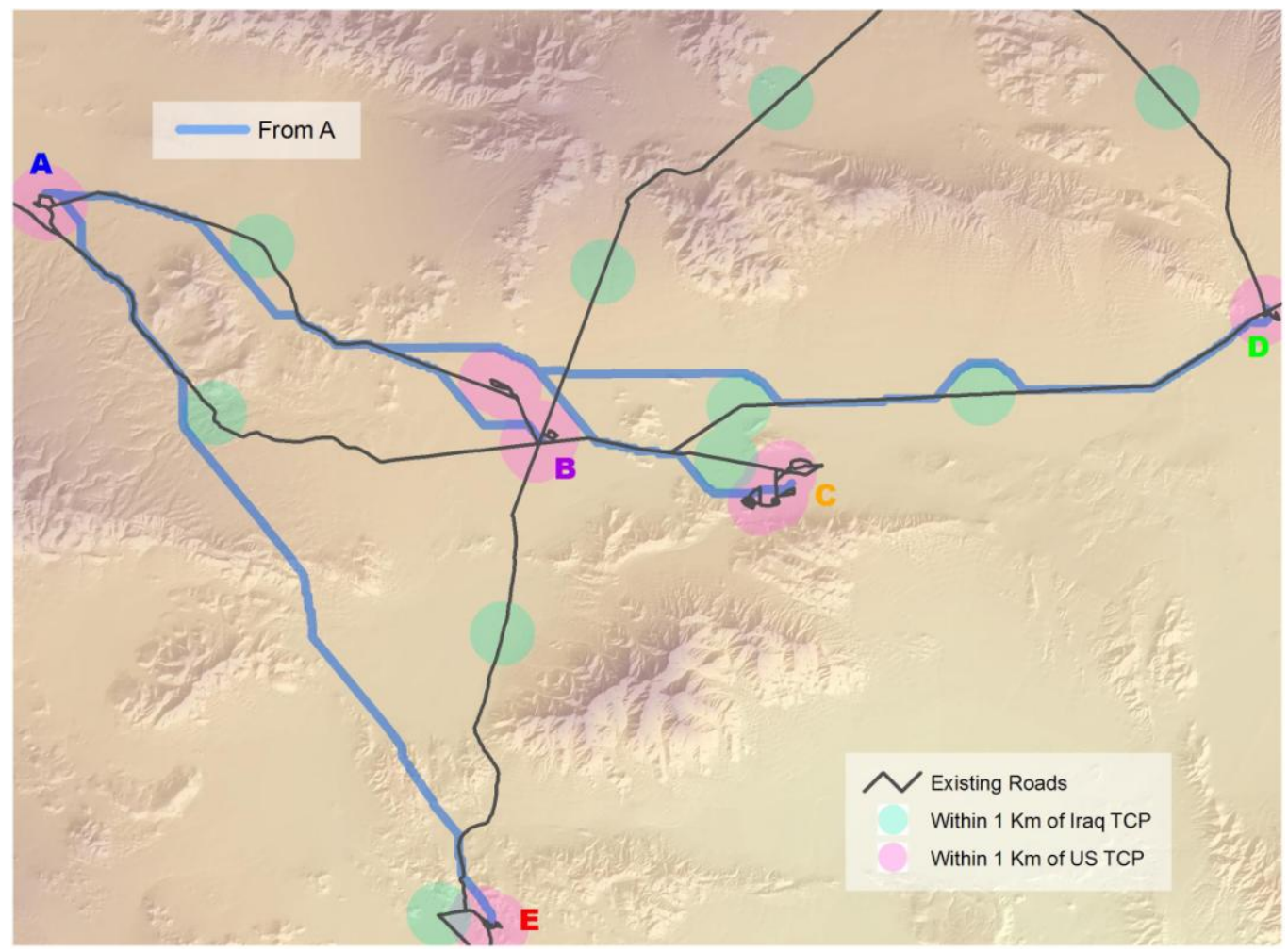

Figure 7-7. Vector-based Layers at 100-meter Resolution

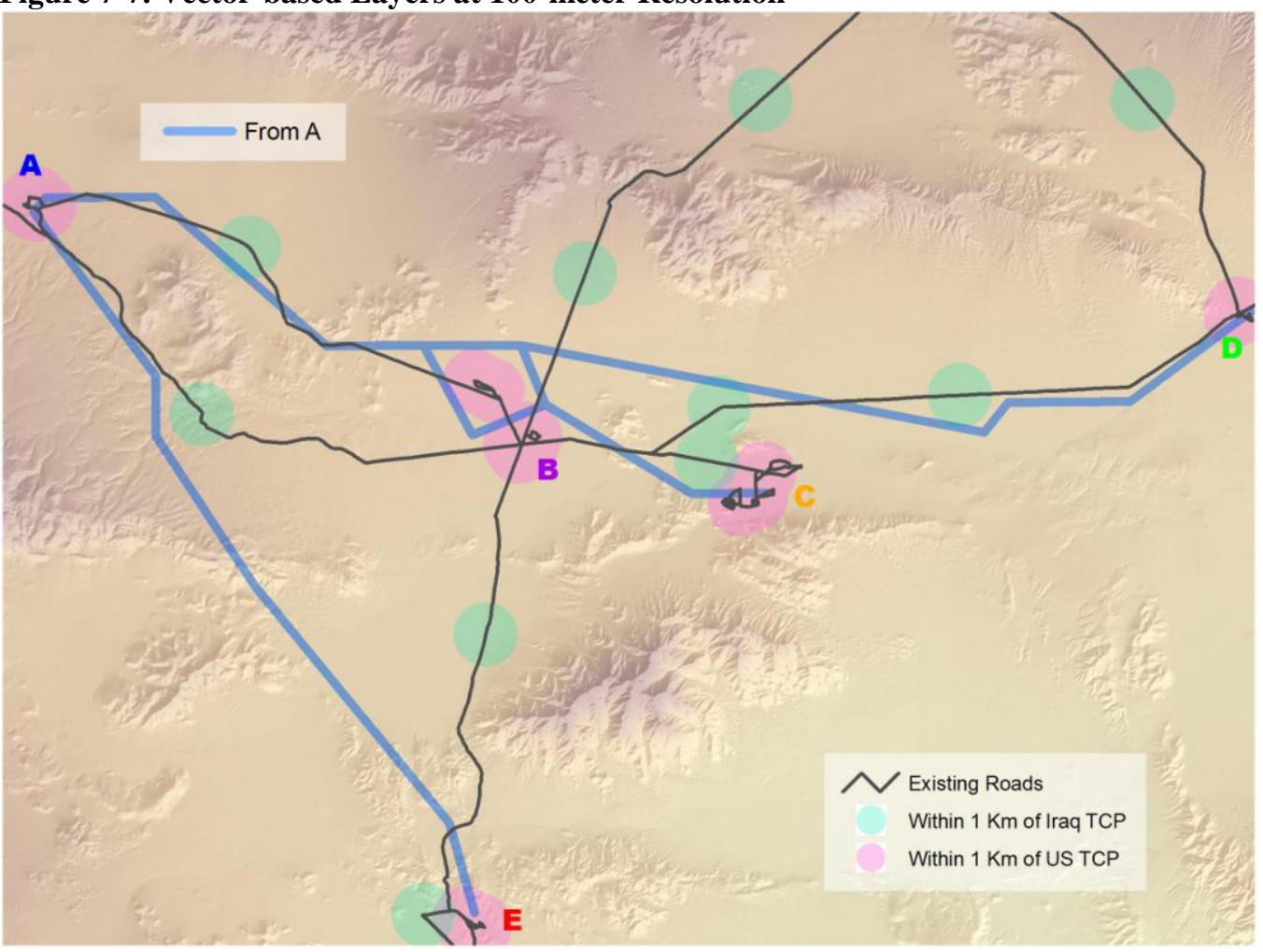

Figure 7-8. Vector-based Layers at 1000-meter Resolution 


\subsection{A New Variable Input}

This section tested different data within the same study area and data from a new geographic location.

\subsubsection{TCP}

A new TCP feature dataclass was created from the original TCP data. The data consisted of the same attributes as the original TCP data that the client provided. The new TCP was renamed TCP2 and was edited, included moving several Iraqi and US checkpoints to new positions. A few of the original checkpoints were also deleted and a few checkpoints were left unchanged.

The weights for both checkpoints were set to eight, while the rest of the variables were set to one. Two tests were completed using different start and end points. The first test was conducted with point $\mathrm{A}$ as the start point and points $\mathrm{B}, \mathrm{C}, \mathrm{D}$, and $\mathrm{E}$ as the end point. The second test used point $\mathrm{E}$ as the start point and points $\mathrm{A}, \mathrm{B}, \mathrm{C}$, and $\mathrm{D}$ as the end point.

The model generated two routes, as expected, based on the results from the model development section. Figure 7-9 illustrates that the route avoided the new checkpoints at 1000 meters. This result illustrated that new spatial datasets within the study area can work in this model.

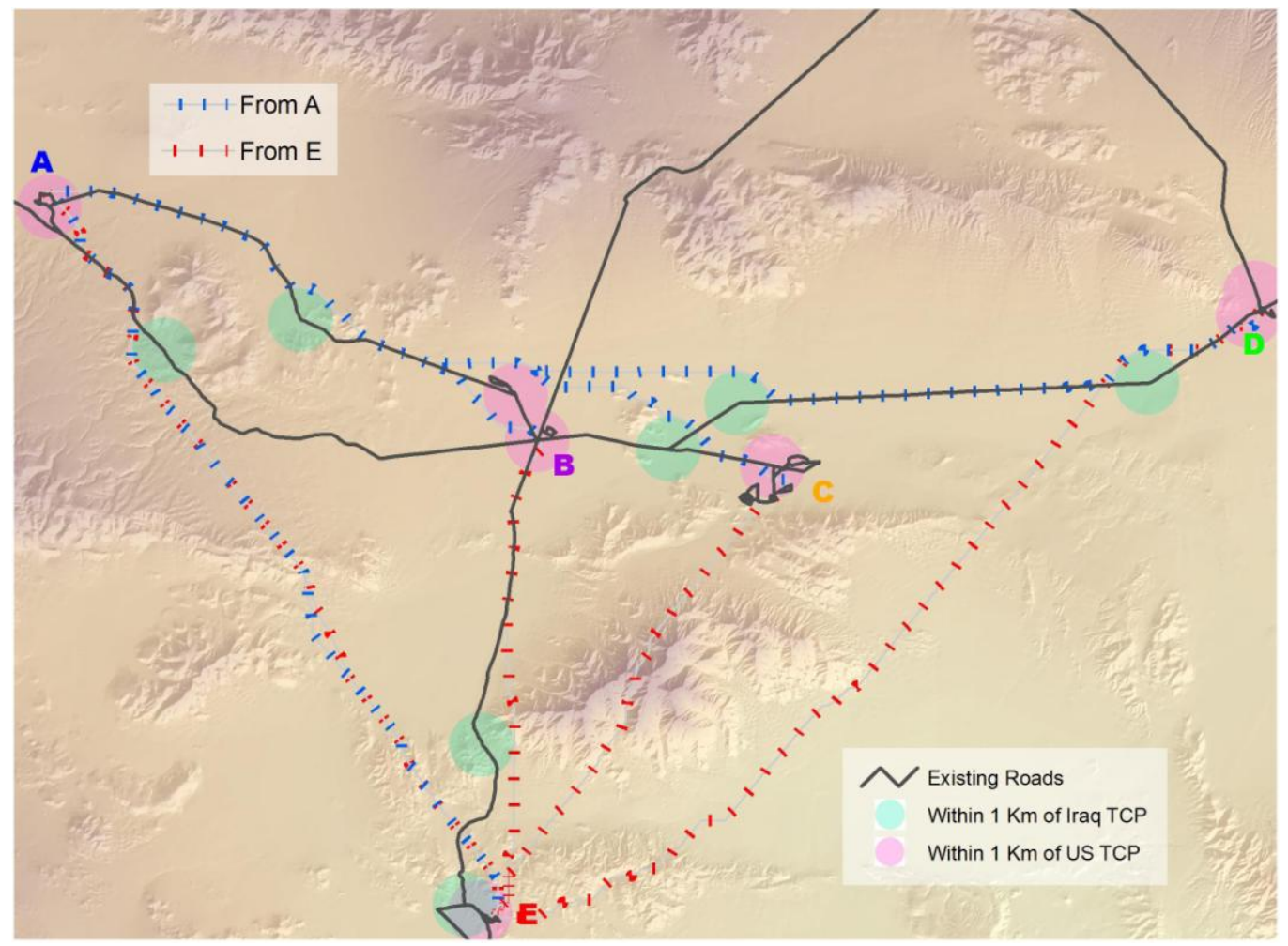

Figure 7-9. New Data Input Test 


\subsubsection{A New Study Area}

To further prove that this model is robust, a new study area was defined. The new study area consisted of data from Lindsay, California, and the surrounding area. The same data inputs were found and used. The DEM had a ten meter resolution and was retrieved from the USGS website. The vector data used the same attribute tables as the data used in the Fort Irwin study area. Road data was created by Tele Atlas and ESRI. The hydrology data was created by the USGS and ESRI. Both datasets were published by ESRI. The TCP, start point, and end point datasets were created specifically for this scenario.

The new datasets were used as the inputs for the model. Two tests were conducted with point $\mathrm{A}$ as the start point and points $\mathrm{B}, \mathrm{C}, \mathrm{D}$, and $\mathrm{E}$ as the end point. The first test used both checkpoint weights set to three and the other three variables set to one. The generated routes correctly avoided the checkpoints by at least 300 meters (see Figure 7$10)$.

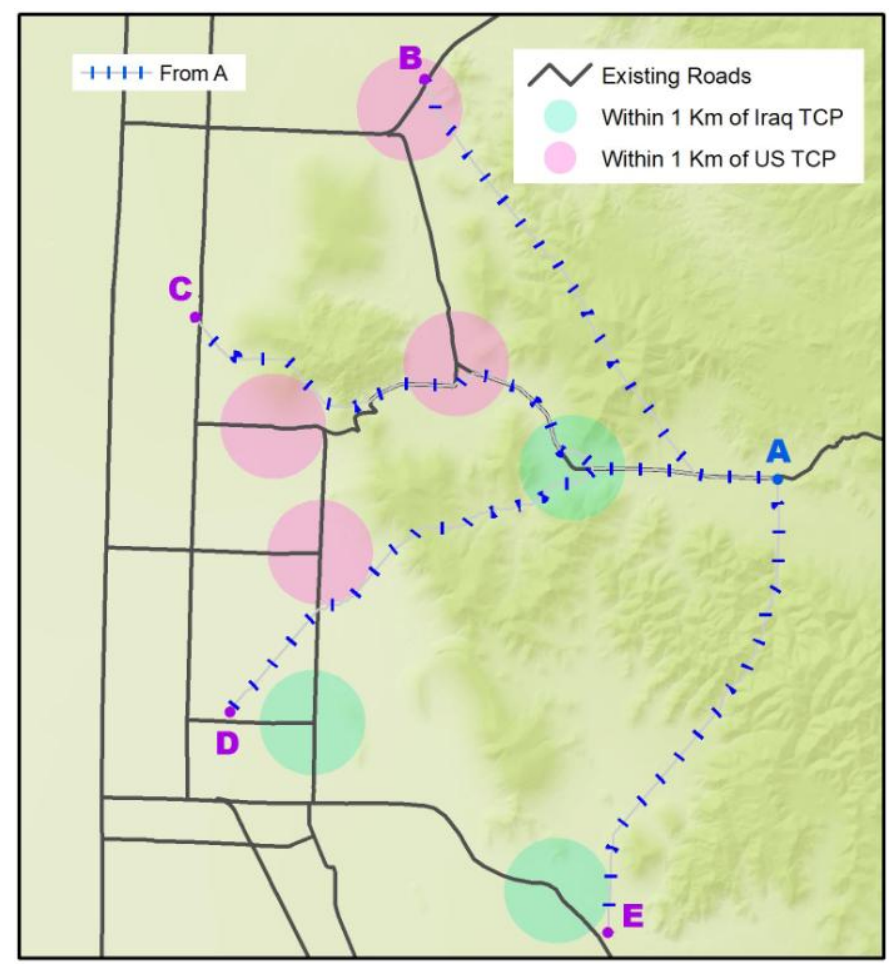

Figure 7-10. First New Study Area Test

The second test changed the checkpoint weight values to eight. The generated routes correctly avoided the checkpoints at 1000 meters (see Figure 7-11). The only exception was the route from point $\mathrm{A}$ to $\mathrm{B}$, which necessarily goes within 1000 meters of the checkpoint because point B is within 1000 meters of a checkpoint. 


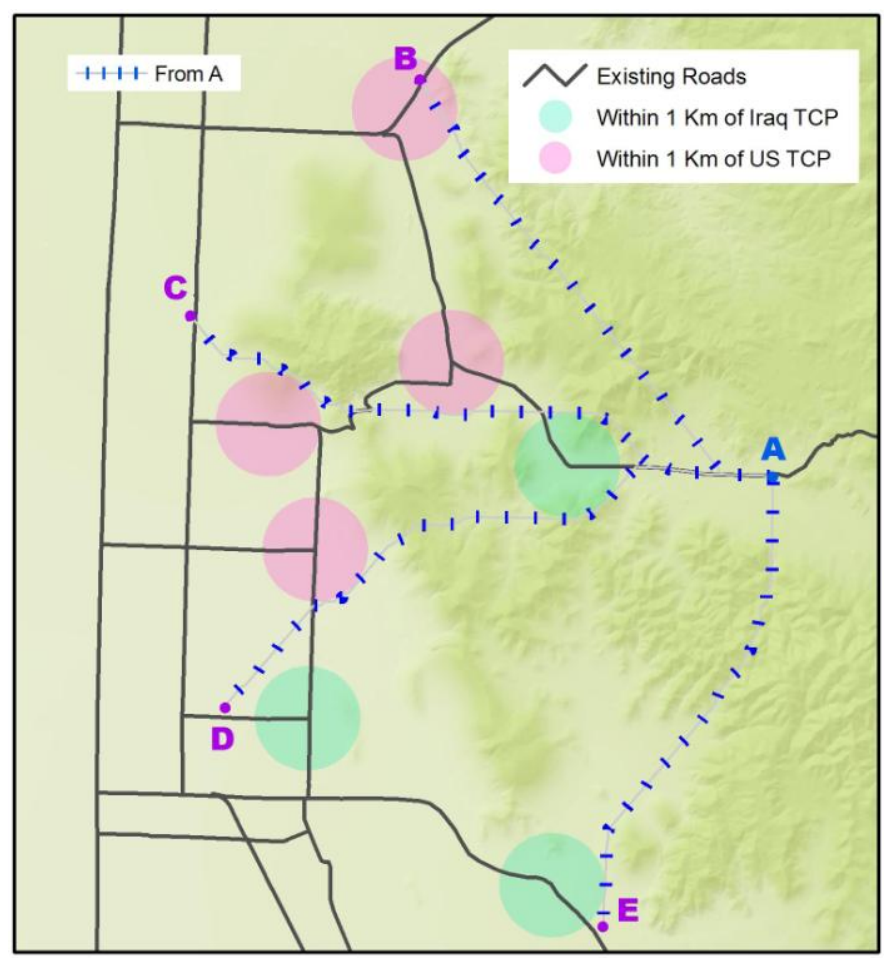

Figure 7-11. Second New Study Area Test

These two tests illustrated that the model is more than a prototype and is versatile in its ability to use data in any study area. The model did not have to be modified to accommodate the new data. Before running the model tool for the new study area, only one environment setting was changed: the extent was changed to match the DEM of the new study area. 


\section{Deliverables}

This project required two main deliverables to the client. The first was a geodatabase that contained the toolbox that held the model. The other deliverable was a user manual that describes how to run the model. These items were delivered on a CD with other useful elements from the project. Before the deliverables were handed over to the client, a test running the model on another computer was conducted.

\subsection{Computer Transition Testing}

To ensure a smooth transition of the model to the client, a test of the model was conducted on another computer by Mr. Russ Johnson, someone familiar with ArcGIS, but unfamiliar with running the Tactical Route Generation Tool model. In November of 2008, a flash drive containing the necessary data and model was loaded onto his laptop computer. A new, empty AcrMap document was created with the Fort Irwin data and model. Following the instructions in the user manual, Mr. Johnson opened the model and created a test scenario between two different MOUT sites. The model ran successfully on his computer. At Mr. Johnson's suggestion, minor revisions were made to the manual for easier transition to the client.

\subsection{Client Deliverables}

All deliverables were provided on a data $\mathrm{CD}$ to the client. The CD contained a folder called RouteTool and had following the items in the folder:

- Fort Irwin file geodatabase

- Results folder

- A User Manual document

- Original Data folder

The Fort Irwin file geodatabase contained all of the data inputs used in the model. There were two feature datasets with the geodatabase and one raster dataset. The input feature dataset consisted all of the base data for the model. The sites feature dataset consisted of the possible start and end points that were selected from the client's info dataset. The geodatabase also contained the DEM as a raster dataset. The final object in the geodatabase was the RouteTool toolbox. This toolbox included the model and a toolset. The model was provided as three separate models within the toolset. This allowed for faster iteration of the model; instead of running the entire model each time to generate a path, these smaller models provided a quick way to rerun the model.

The results folder contained two geodatabases, one called results and one called finalroutes. The results geodatabase is designed to contain all intermediate data from the model, which is stored for possible future use by the client. The results geodatabase was empty on delivery to the client. The finalroutes geobasedatabase contained the routes that were produced in the model development and testing sections, and could be used to store future model output.

A user manual document was created to instruct the client on how to use the model. The user manual can be found at the end of this document as appendix 2 . 
The last item on the $\mathrm{CD}$ was the original data folder, which contained the data the client provided for this project. 


\section{Conclusion}

This project demonstrated how to create plausible off-road routes that the client could use in a simulated environment. The generated route output, in a shapefile format, meets the shapefile requirement this is compatible with the VBS2 software.

The generate routes from the test scenarios were best analyzed for avoiding checkpoints between MOUT sites. Avoiding checkpoints within a MOUT site was not analyzed extensively. The original TCP dataset contained a cluster of checkpoints at each MOUT site, which made the route difficult to avoid those checkpoints. There may also be other factors or physical features within each site that were not used to influence the generated route. One example is the presence of buildings.

The initial concept of this project was to build a customized tool to be applied to a specific study area. The model was demonstrated to be adaptable to any set of data, not only within the study area, but also with an entirely new collection of datasets for a different geographical location.

\subsection{Limitation}

One major limitation of this project deals with determining the usable slope values. The reclassification of the slope raster was based on the capabilities of a HMMWV, which has specific uphill slope and cross slope ratings (see section 5.3.1). The model used only the lesser of the two ratings. Additionally, the slope raster contained only a single value for each cell in the DEM. This meant that the slope was only accounted for in one direction, and the model was not considering whether the route was uphill, downhill, or across the slope.

\subsection{Recommendations}

The model can be improved in a few ways. Recommendations include incorporating post-model tasks, adding more variables, testing more scenarios, incorporating different vehicles, and creating a new user interface.

There are two post-model tasks that need to be completed before loading the new data into the VBS2 software. However, these tasks were beyond the scope of this project, but are described here. The first task is to edit the new off-road path. Parts of the path followed where there is an existing road and needs to be deleted. The new road data should be added as a layer into an ArcMap document. An edit session is needed to edit the road feature class. The off-road path will be selected and the Split tool will be used at where the off-road path overlapped the existing roads. The Split tool creates segments of the off-road and those segments that are overlapping the existing roads need to be deleted. The remaining off-road path segments end vertices need to be snapped to the existing road. This is all the editing that needs to be done with the off-road path. The final task is to convert the data into a shapefile. In ArcMap, the road layer can be selected. A right click on the layer allows for a menu to appear. The menu contains data as a selection and another menu appeared. The export data option should be used to convert the data into a shapefile (see Figure 9-1). It is recommended that these post-model tasks are to be incorporated into the model for better efficiency. 


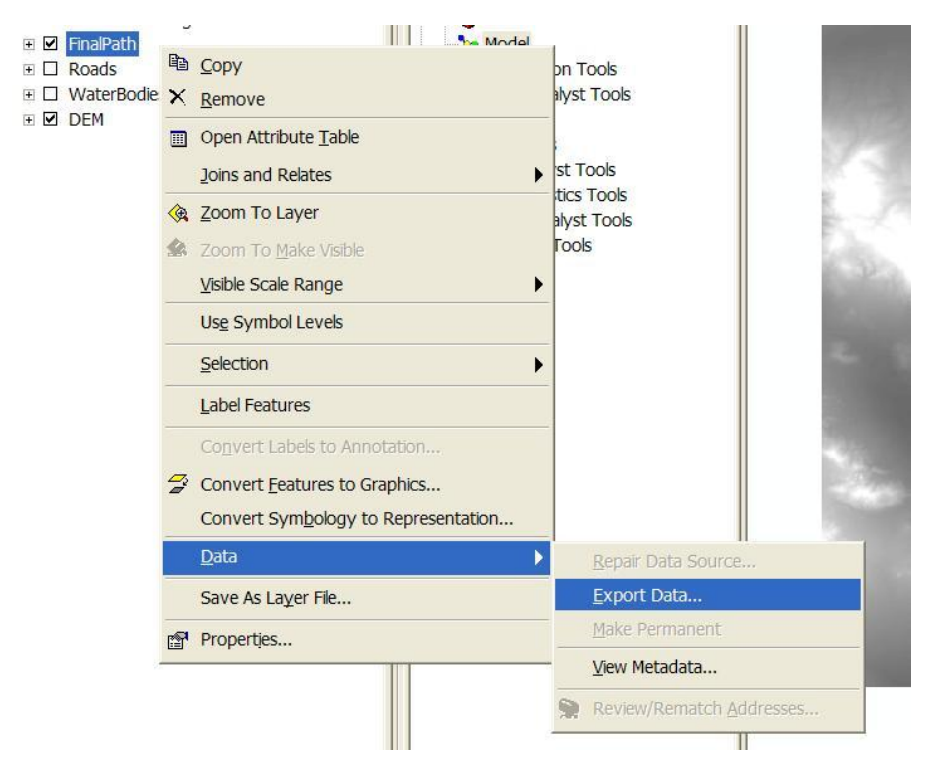

Figure 9-1. Converting Data Into A Shapefile

Least cost path analysis moving across the terrain usually uses more variables. Soil and vegetation data are two of the more commonly used factors. The client did not provide that data. However, the client did provide other datasets that were not used including buildings and vertical ridges. These datasets could be incorporated into the model.

This project used only integers for the new rasters that were created from the Reclassification and Weight Sum tools. Using floating point values may provide more realistic results. A floating point value is any numeric value with a decimal point. Since the slope is continuous, using floating point should be considered. However, the output of the reclassify tool will always be in integer format. The Weighted Sum tool can use floating values for the weight field.

Limited scenario testing was done on the data. Changing each of the reclassified rasters can be conducted to test for more accurate scenarios. This may be necessary to reflect new situations. For instance, insurgents may be avoiding checkpoints at a greater distance. If so, then changing the buffer distance to represent this change would be necessary.

Another modification to the model to increase versatility is the ability to incorporate different vehicle types. Reclassifying the slope raster would generate a route for a different vehicle. Other military vehicles considered include HETs, Bradleys and Abrams. Sedans, pickups trucks, and cargo vehicles are the civilian vehicles of interest for this project.

Programming an extension for this model would help provide a more comprehensive interface to easily modify any of the individual tools. An extension might include optional software compatible with ArcGIS software. Generally, an extension contains customized tools and contributions to the functionally to the software. A customized extension could include a user-friendly interface allowing quick access to changing different settings throughout the model tool. The ability to change the reclassified slope raster to accommodate another vehicle type through an interface is more desirable than editing the model. 


\section{References}

Balstrom, T. (2002). On identifying the most time-saving walking route in a trackless mountainous terrain. Danish Journal of Geography, 102, 51-58.

Collischonn, W., \& Pilar, J. V. (2000). A direction dependent least-cost-path algorithm for roads and canals. International Journal of Geographical Information Science, 14(4), 397-406.

Crawford, M. A. (2008). An analysis of terrain roughness: generating a gis application for prescribed burning. Texas Tech University. Retrieved November 11, 2008, from http://etd.lib.ttu.edu/theses/available/etd-04022008102323/unrestricted/Crawford_Matthew_Thesis.pdf

Donlon, J. J., \& Forbus, K. D. (1999). Using a geographic information system for qualitative spatial reasoning about trafficability. Paper presented at the Proceedings of the 13th International Workshop on Qualitative Reasoning; June 1999; Lock Awe, Scotland.

Eichrodt, A. W. (2003). Development of a spatial trafficability evaluation system [dissertation]. Zurich, Switzerland: Swiss Federal Institute of Technology Zurich. 184 p.

ESRI (2008). ArcGIS 9.3 Desktop Help. Retrieved October 15, 2008, from http://webhelp.esri.com/arcgisdesktop/9.3/index.cfm?TopicName=Cost_Distance

Goodchild, M. F. (2000). GIS and transportation: status and challenges. GeoInformatica, $4(2), 1127-1139$.

Hoffman, R., \& Krotkov, E. (1989). Terrain roughness measurement from elevation maps. Society of Photographic Instrumentation Engineers, Mobile Robots IV, $1195,104-114$.

Lee, J., \& Stucky, D. (1998). On applying viewshed analysis for determining least-costpaths on digital elevation models. International Journal of Geographical Information Science, 12(8), 891-905.

Moreno, M., Levachkine, S., Torres, M., \& Quintero, R. (2003). Geomorphometric analysis of raster image data to detect terrain ruggedness and drainage density, Lecture Notes in Computer Science (Vol. 2905, pp. 643-650).

Slocum, K. R., Surdu, J. R., Sullivan, J., Rudak, M., Colvin, N., \& Gates, C. (2002). Trafficability Analysis Engine. The Journal of Defense Software Engineering. June 2003

Suvinen, A., Saarilahati, M., \& Tokola, T. (2003). Terrain mobility model and determination of optimal off-road route. In: ScanGIS 2003 Proceedings of the 9th Scandinavian Research Conference on Geographical Information Science. pp. 251-259.

Tobler, W. (1993). Three presentations on geographical analysis and modeling technical Report 93-1). Santa Barbara, California: University of California, Natioanl Center 
For Geographical Information and Analysis. Retrieved February 11, 2008, from http://www.ncgia.ucsb.edu/Publications/Tech_Reports/93/93-1.PDF

TRADOC, (2008). About TRADOC. Retrieve October 13, 2008 from http://www.tradoc.army.mil/about.htm

Wood, B. M., \& Wood, Z. J. (2006). Energetically optimal travel across terrain: visualizations and a new metric of geographic distance with archaeological applications. Retrieved Februrary 11, 2008 from http://users.csc.calpoly.edu/ Zwood/research/pubs/bwoodSPIE06.pdf

Yu, C., Lee, J., \& Munro-Stasiuk, M. J. (2003). Research article: extensions to least-costpath algorithms for roadway planning. International Journal of Geographical Information Science, 17(4), 361-376. 


\section{Appendix A - Tool Reference}

The model tool was constructed by stringing together several individual tools from existing toolsets contained in different ArcToolboxes. Appendix Table 1A contains the individual tool name and the location of where it originally came from. Several tools were used more than once. If a tool was used more than once, the amount of times it was used is specified after the tool name.

\begin{tabular}{l|ll}
\hline \multicolumn{2}{l}{ Appendix Table1A } \\
\hline Individual Tool (times used) & ArcToolbox & Toolset \\
\hline Add Field (3) & Data Management Tools & Fields \\
Calculate Field (3) & Data Management Tools & Fields \\
Cost Distance & Spatial Analyst Tools & Distance \\
Cost Path & Spatial Analyst Tools & Distance \\
Feature to Raster (4) & Conversion Tools & To Raster \\
Merge & Data Management Tools & General \\
Multiple Ring Buffer & Analysis Tools & Proximity \\
Raster to Polyline & Conversion Tools & From Raster \\
Reclassify (5) & Spatial Analyst Tools & Reclass \\
Select (2) & Analysis Tools & Extract \\
Slope & Spatial Analyst Tools & Surface \\
Weighted Sum & Spatial Analyst Tools & Overlay \\
\hline
\end{tabular}




\section{Appendix B - User Manual}

This appendix was designed to illustrate how to implement the tactical route generation tool onto the clients' computer system.

\section{Copy Folders}

1. Insert the CD into the computer.

2. Open the folder and copy the RouteTool folder to the $\mathrm{C}$ drive.

\section{Using ArcMap}

1. Open ArcMap. Either open an existing map document that contains all of the data needed to run the tool or open the FortIrwin.mxd found at

C:IRouteToollFortIrwin.mxd

2. Add the following data if not present
a. DEM
b. Roads
c. TCP
d. Water bodies
e. Start Point
f. End point

3. Edit data to the following standards

a. DEM - This cannot be in DTED format.

b. Roads - This must be a linear geometry.

c. TCP - Make sure there is an attribute field title "TCP" and the only two valid entries are either "IRQ" or "US". For future uses, only "US" will be necessary and all other entries will be considered non-US.

d. Water bodies - This must have a polygon geometry.

e. Start Point - This must have a point geometry. The start point can contain more than one point, but only one point is recommended.

f. End Point - This mush have a point geometry. The end point can have multiple points as long as the start point is not contained in this layer.

g. All data should be in the same projection.

4. Adding the Tactical Route Generation Tool

a. If the ArcToolbox window is not present, then click the icon on the standard toolbar to show ArcToolbox.

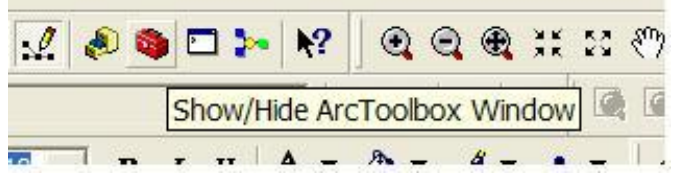

b. Right click in the white space of the ArcToolbox window and click Add Toolbox. 


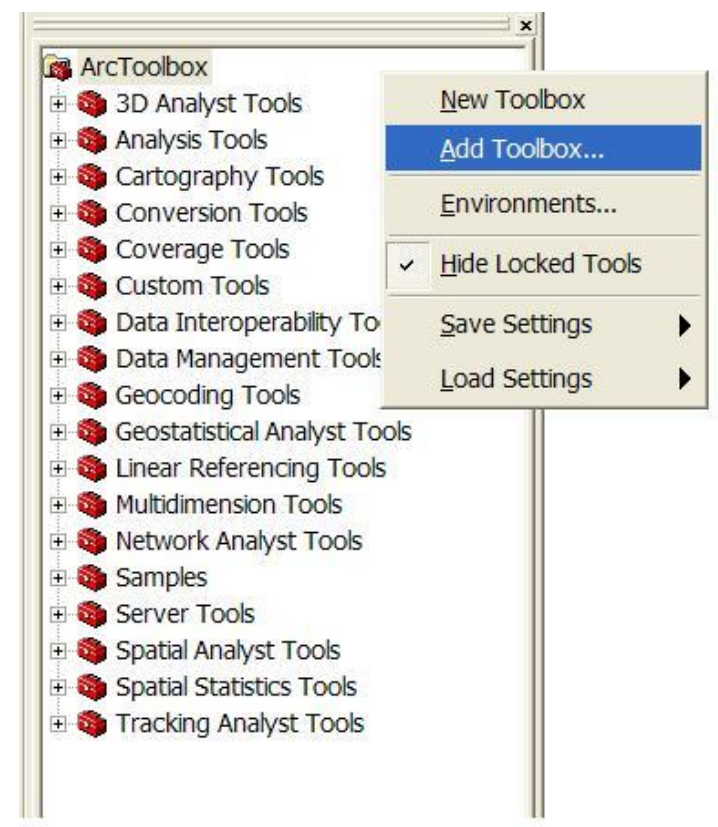

c. A dialog box will appear to add a toolbox. Navigate to

$\mathrm{C}:$ RouteToollFortIrwin.gdb and left click once on the RouteTool toolbox. Click open.

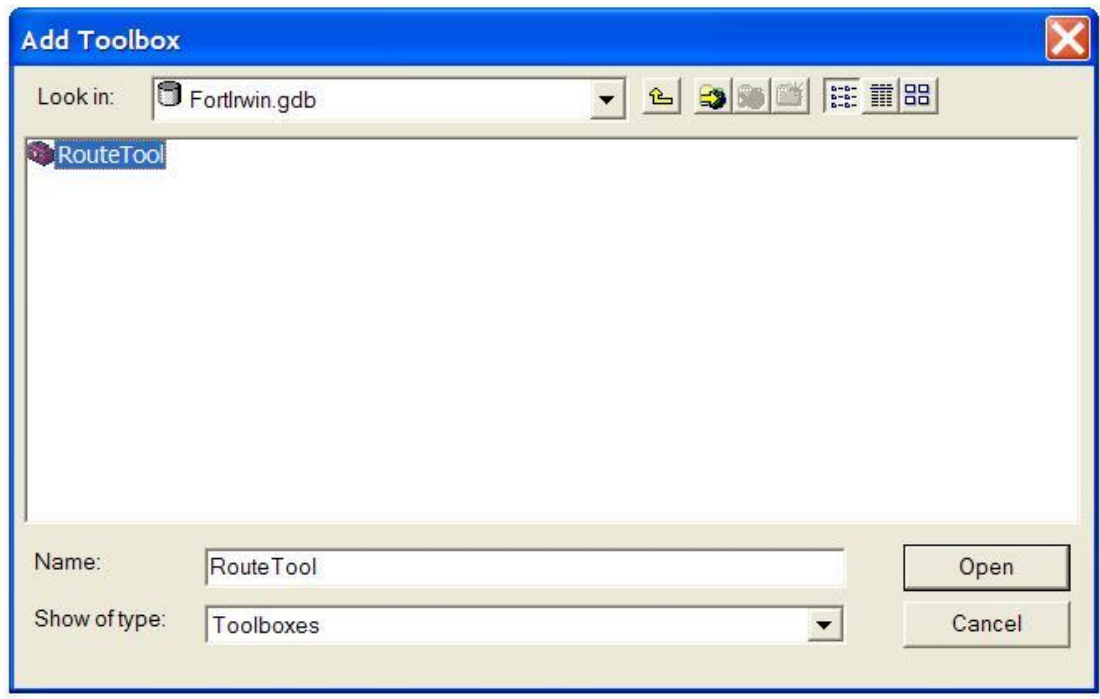

5. Running The Model

a. Then RouteTool toolbox will be added alphabetically to the ArcToolbox window. Expand the tool by clicking on the plus sign next to the name. Right click on the HMMWVRoute model and click open. 


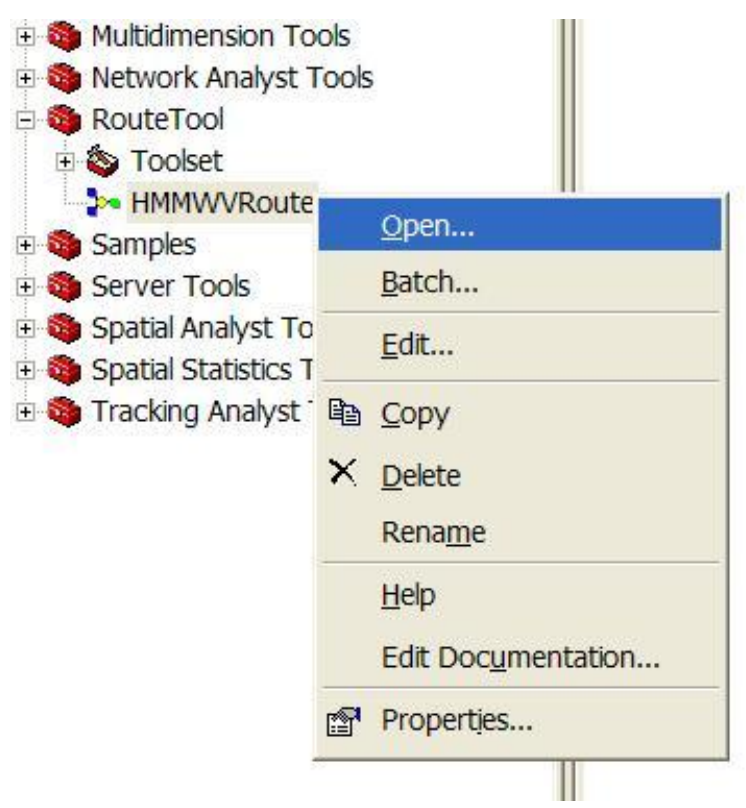

b. The model interface will appear. First click on the environments button. In the environments dialog box, under the general settings, set the extent to the DEM that will be used. Select ok to close the environment settings dialog box.

Select the appropriate data for each parameter. The last parameter is the name and file path to where the generate route(s) will be placed. Pick a name and location with a geodatabase for the generate route to be placed in. Click ok on the HMMWVRoute dialog box.

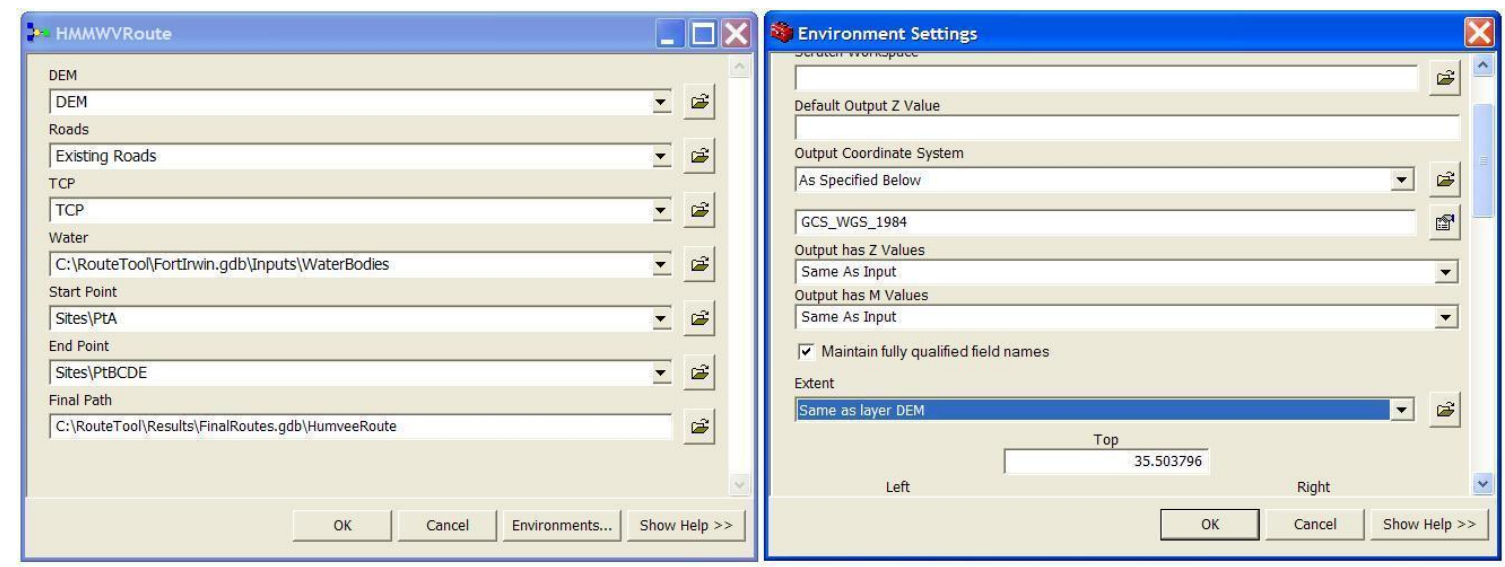

6. Post-Editing Tasks

a. The model will run and the results will be added to the map document. Double click on the new layer and select the symbology tab in the layer properties dialog box. On the left hand side select Unique values under Categories. Select Type for the Value Field. Click Add All Values button. Chose two different symbols to distinguish the Offroad (new paths that avoid the checkpoints) from the existing roads (value $=$ null). Click ok. 


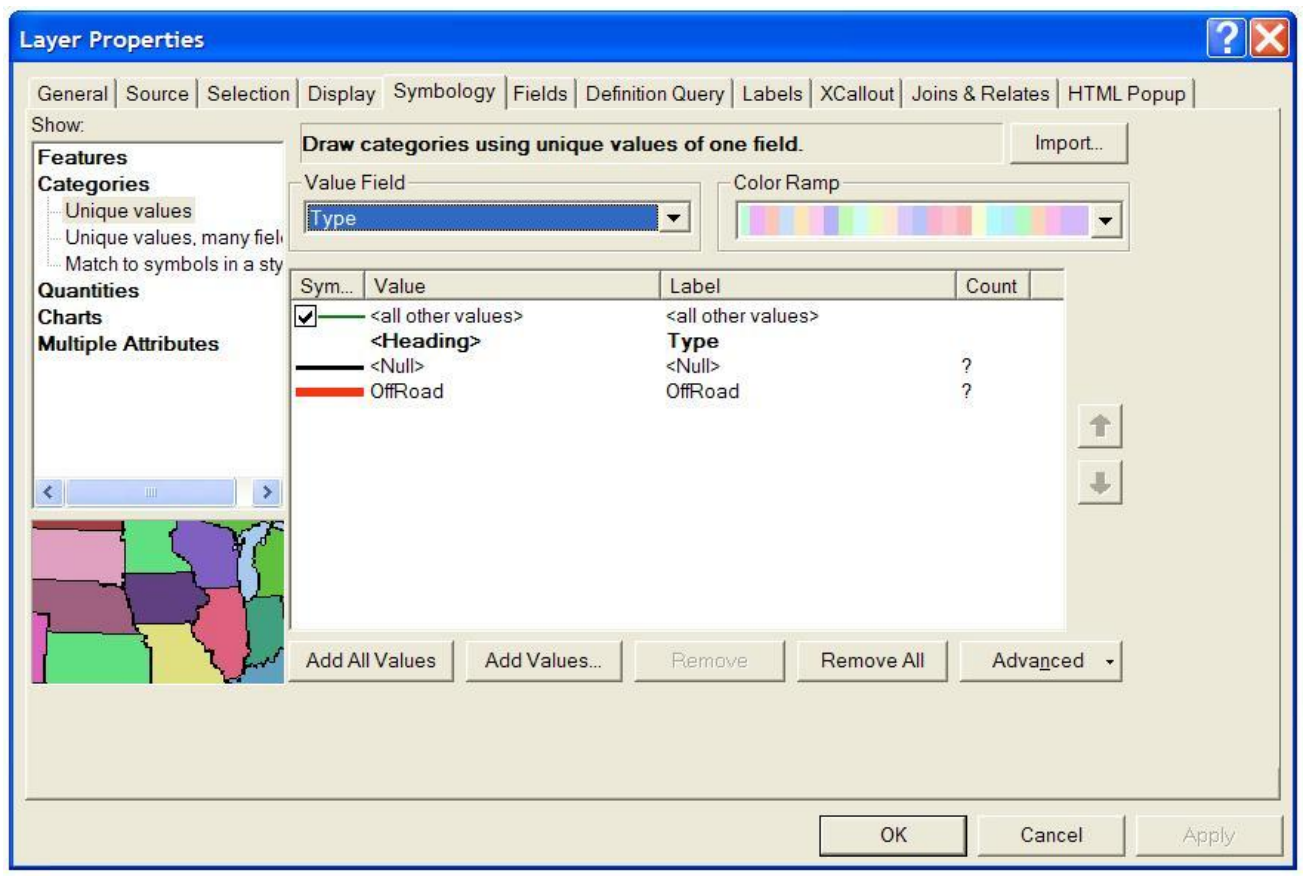

b. Perform the necessary edits needed to be compatible with the VBS2 software. This should include deleting the portions of the Offroad path that coincide with the existing roads. The new segments should then have the end vertices snap to the existing roads.

c. After the edits have been made, right click the layer in the table of contents. Select Data from the options available and click on the Export Data option.

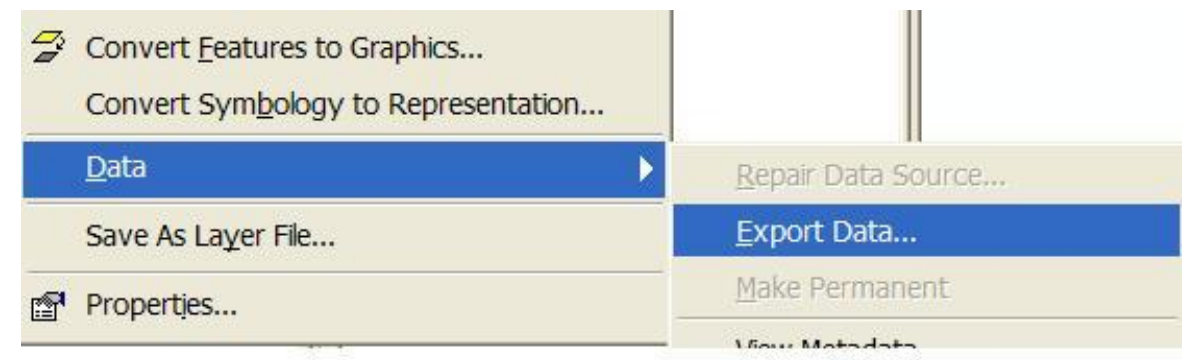

d. The Export Data dialog box will appear. In the output shapefile or festureclass click the browse button. Designate a name and file path the where the data will be exported to. Under Save as type, make sure it is a shapefile. 


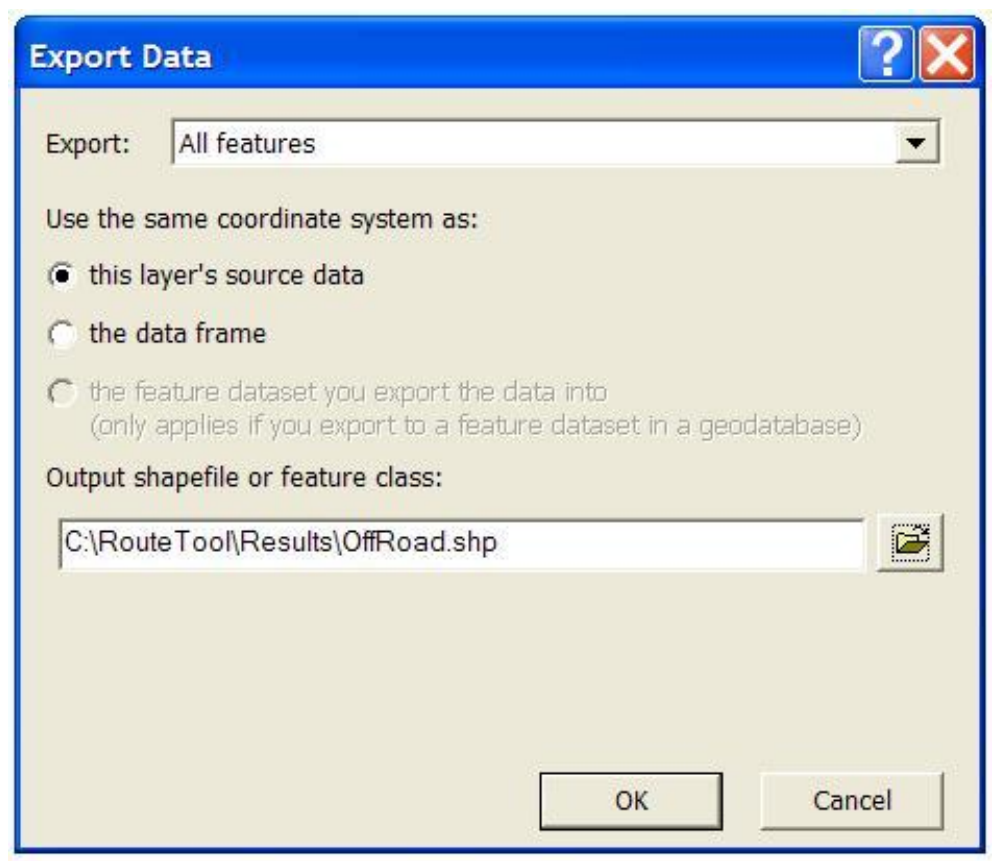

e. The data is now ready to be used for the VBS2 software.

\section{Using the Three Separate Models}

The separate models can be found in the toolset. These models are direct portion copies from the original model. These models should be used in the following order:

1. Reclassified Rasters

This first model requires the DEM, roads, waterbodies, and TCP features as the inputs. The outputs will be placed in the results geodatabase under the following names: DEMrecl, Roadrecl, WtrRecl, IRQrecl, and USrecl.

2. Weight Sum

This second model takes the reclassified rasters and creates a cumulative cost surface. The inputs that are required are the DEMrecl, Roadrecl, WtrRecl, IRQrecl, and USrecl. The output will be placed in the results geodatabase under the name WeiSum. The default weights are set to one. These weights should be changed before running the model.

3. LCP and Software Compatibility

This third model takes the cost surface with start and end points and creates the final route. Additional steps are also taken to have the final result become compatible with VBS2 software. The inputs that are required are the WeiSum (Cost Surface), start point, end point, and existing roads. The final output will be defined by the user.

\section{Modifying the Model}


The model may need to be modified to fit different or future scenarios. Here are a few recommendations on how to modify the model. Please note that only the first modification has been tested.

To modify any of the individual tools in the model, right click the HMMWVRoute model and click on edit. This opens up the ModelBuilder dialog box.

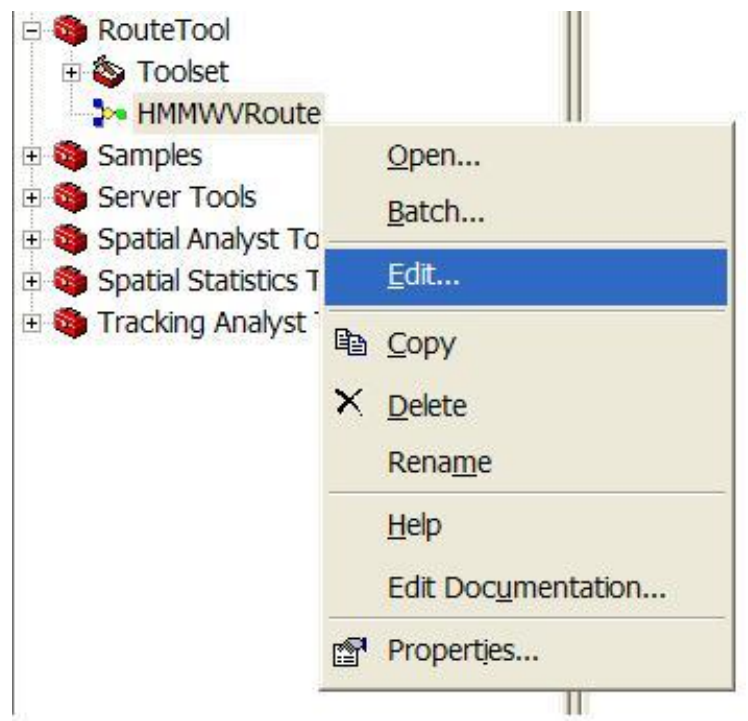

In the ModelBuilder dialog box, select the tool (yellow boxes) that you wish to modify. The following tools include reasons and recommendations for modifications.

\section{Weight Sum}

The weight sum tool affects the how the route will be generated. There are five weights that are associated with the variables to make the cost surface. The hydrology weight is the only value that will not change. The Iraqi and US buffer checkpoints were the two most commonly changed values. In order for the generated route to avoid at checkpoints at certain distance, those values were changed. When the values were at 3 , the route avoided most checkpoints at a distance of 300 meters. When the values were at 8 , the route avoided the checkpoints at a distance of 1000 meters. Changing the DEM and slope weight values will also change the route, but these changes were not heavily tested.

2. Reclassifications

Each variable was reclassified.

Slope - This was derived from the DEM. Changing the reclassifications should only be done if the route is intended for a different vehicle.

Roads - Reclassification should only be done if the weight for the area of the non-existing roads should be changed.

Hydrology - Reclassification should only be done if there is water that is passable by the vehicle but show still be avoided. 
Checkpoints - There are two sets of checkpoints derived from the TCP data. If the amount of influence either the Iraqi or US checkpoints has changed on the path an insurgent might take, then he reclassifications should be done modified to reflect that influence.

\section{Selecting Checkpoint}

The select tool that selects the non-US TCP points will have to be redefined for the data from a new study that contain different TCP attribute values. For example, the select tool for the non-US TCP points should read "TCP not equal to 'US"”

\section{Buffer Distances}

The multiple buffer ring tool was set at distances of the effect weapon range from each checkpoint. These ranges effect how close an insurgent would get to a checkpoint. If the effect weapons change or any other factor that would contribute to having a insurgent avoid a checkpoint, modifications to the buffer distance is recommended to reflect those changes. If a change to the buffer is made, those same changes must be reflected in the reclassify tool. 